\title{
Enantioselective Synthesis of Ferrocenyl Nucleoside Analogs with Apoptosis-Inducing Activity
}

\author{
Philippe James $^{\dagger}$, Jörg Neudörfl ${ }^{\dagger}$, Moritz Eißmann ${ }^{\ddagger}$, Patrick Jesse ${ }^{\ddagger}$, Aram Prokop ${ }^{\ddagger}$, and \\ Hans-Günther Schmalz ${ }^{\dagger *}$ \\ ${ }^{\dagger}$ Institute of Organic Chemistry, University of Cologne, Greinstr. 4, 50939 Köln, Germany \\ ${ }^{\ddagger}$ Department of Pediatric Oncology/Hermatology, University Medical Center Charité, \\ Campus Virchow, Humboldt University of Berlin, 13353 Berlin, Germany \\ schmalz@uni-koeln.de
}

\section{Supporting Information}

Contents: experimental procedures and full characterization of new compounds (6-11, 1318); X-ray crystallographic data for compounds 6 and 15; ${ }^{1} \mathrm{H}$ and ${ }^{13} \mathrm{C}$ NMR spectra.

\section{General experimental conditions}

Reactions were conducted in flame-dried glassware under an atmosphere of argon using freshly distilled anhydrous solvents. NMR spectra were recorded at $300 \mathrm{MHz}$ for protons and at $75 \mathrm{MHz}$ for carbons. Deuterated chloroform was used as solvent unless otherwise indicated. Proton shifts are reported in ppm ( $\delta$ ) downfield from TMS and were determined by reference to the residual solvent peaks $\left(\mathrm{CDCl}_{3}: 7.24 \mathrm{ppm}, \mathrm{CD}_{3} \mathrm{OD}: 3.31 \mathrm{ppm}\right)$. Data are reported as follows: chemical shift (multiplicity [singlet (s), doublet (d), triplet (t), quartet (q), quintet (quint), hexet (hex) and multiplet (m)], coupling constants [Hz], integration, assignment). ${ }^{13} \mathrm{C}$ NMR spectra were recorded using an APT sequence with complete proton decoupling. Multiplicities $\left(\mathrm{C}, \mathrm{CH}_{2}\right.$ or $\left.\mathrm{CH}, \mathrm{CH}_{3}\right)$ were deduced from these spectra. ${ }^{13} \mathrm{C}$ chemical shifts are reported in ppm $(\delta)$ relative to solvent resonance as the internal standard $\left(\mathrm{CDCl}_{3}: 77 \mathrm{ppm}, \mathrm{CD}_{3} \mathrm{OD}: 49.05 \mathrm{ppm}\right)$. Melting points are not corrected. Optical rotations were recorded at the given wavelengths (path length $100 \mathrm{~mm}$ ).

The TMS-protected nucleobases (TMS) $)_{2}$ cytosine and (TMS) $)_{2}$ uracil, respectively, were prepared from cytosine or uracil by treatment with an excess of HMDS and TMSCl at $80^{\circ} \mathrm{C}$ for $2 \mathrm{~d}$ and isolation of the product by distillation under reduced pressure ( 0.1 torr). 


\section{Synthetic procedures and characterization of the new products}

\section{II.1. Synthesis of ester 5 through diastereoselective ortho-deprotonation/alkylation.}

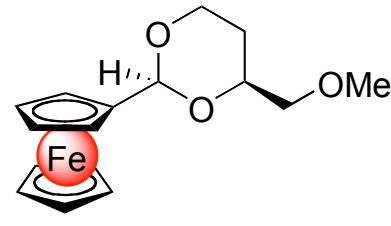

4

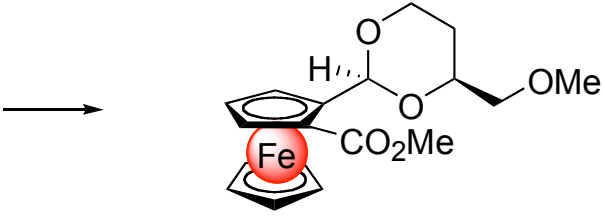

5

To a cooled solution of $\mathbf{4}^{1}\left([\alpha]^{20}{ }_{\mathrm{D}}-32.4, c 0.70, \mathrm{CHCl}_{3},[\alpha]_{\mathrm{D}(l i t .)}{ }^{1}-32.5 ; 4.03 \mathrm{~g}, 1\right.$ equiv., $12.75 \mathrm{mmol})$ in dry diethyl ether $(65 \mathrm{~mL})$ under argon atmosphere was added dropwise at $78^{\circ} \mathrm{C}$ a $1.5 \mathrm{M}$ solution of $t$-BuLi in hexanes $(9.4 \mathrm{~mL}, 1.1$ equiv., $14.02 \mathrm{mmol})$. After $10 \mathrm{~min}$. at this temperature, the cooling bath was removed and the temperature was allowed to warm to room temperature and stirred $1 \mathrm{~h}$. The suspension was then cannulated to an other apparatus in an additional funnel which was fixed onto a three-necked round-bottom flask (with thermometer and line to argon/vacuum) containing an excess of methyl chloroformate (9.9 mL, 10 equiv., $128 \mathrm{mmol})$ in ether $(10 \mathrm{~mL})$ maintained at a temperature of $-50^{\circ} \mathrm{C}$. The suspension was then added dropwise slowly to the stirred solution of electrophile. After the addition, the temperature was allowed to warm slowly to room temperature and was stirred overnight at this temperature. The mixture was then quenched with water and the layers were separated. The organic layer was washed with saturated aqueous $\mathrm{NH}_{4} \mathrm{Cl}$ solution, brine, dried over $\mathrm{Na}_{2} \mathrm{SO}_{4}$, filtered and concentrated under vacuum. Flash chromatography (cyclohexane/ethyl acetate $2: 1)$ gave the desired ester $5^{1}$ as a brown oil $\left(3.27 \mathrm{~g}, 69 \%\right.$; $[\alpha]^{20}{ }_{\mathrm{D}}$ $\left.+19.9, c 0.53, \mathrm{CHCl}_{3},[\alpha]^{20}{ }_{\mathrm{D}(\text { lit. })}^{1}+9.0\right)$.

\section{II.2. Synthesis of ester 6 through Horner olefination.}

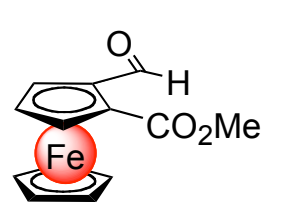

A

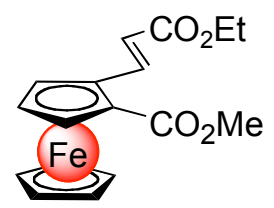

6

The aldehyde A was obtained from $\mathbf{5}$ through acidic acetal cleavage according to Kagan's procedure $^{1}\left([\alpha]_{\mathrm{D}}^{20}+935, c 0.355, \mathrm{EtOH}_{\mathrm{abs}},[\alpha]_{\mathrm{D}(\text { lit. })}^{1}+765,[\alpha]_{\mathrm{D}(\text { lit. })}^{20}-759,(R)\right.$-config. $)$. Transformation of $\mathbf{A}$ into the diester $\mathbf{6}$ was achieved as follow. To a suspension of $\mathrm{NaH}(60 \%$

\footnotetext{
${ }^{1}$ Riant, O.; Samuel, O.; Flessner, T.; Taudien, S .; Kagan, H. B. J. Org. Chem. 1997, 62, 6733.

${ }^{2}$ Rapic, V.; Schlögl, K.; Steinitz, B. Monatsh. Chem. 1977, 108, 767.
} 
in mineral oil, washed with dry hexane, $529 \mathrm{mg}, 1.5$ equiv., $13.21 \mathrm{mmol})$ in THF (37 mL) under argon atmosphere was added at $0^{\circ} \mathrm{C}$, triethylphosphonoacetate $(2.6 \mathrm{~mL}, 1.5$ equiv., $13.21 \mathrm{mmol}$ ) and the resulting mixture was allowed to warm to room temperature and stirred 1 h. After this period, the aldehyde $\mathbf{A}(2.4 \mathrm{~g}, 1$ equiv., $8.81 \mathrm{mmol})$ dissolved in THF $(16 \mathrm{~mL})$ was added to the mixture. The reaction mixture was stirred at room temperature for another 30 min and the reaction was quenched with a solution of saturated $\mathrm{NH}_{4} \mathrm{Cl}$. The layers were separated and the aqueous layer was extracted with EtOAc. The combined organic layers were washed with brine, dried over $\mathrm{Na}_{2} \mathrm{SO}_{4}$ and filtered. Evaporation of the solvents and purification by flash chromatography (cyclohexane/ethyl acetate 3/1) of the residue afforded a dark red oil which solidifies $\left(2.5 \mathrm{~g}, 83 \%\right.$ yield). $\mathbf{M p}\left(\mathrm{CH}_{2} \mathrm{Cl}_{2}\right)+84^{\circ} \mathrm{C} .[\mathbf{\alpha}]^{\mathbf{2 0}}{ }_{\mathbf{D}}+1297.0$ (c 0.41, $\mathrm{CHCl}_{3}$ ). IR (neat) $v 3095(\mathrm{w}), 2978(\mathrm{~m}), 2948(\mathrm{~m}), 2902$ (w), $1711(\mathrm{C}=$ Ost, s), $1694(\mathrm{C}=$ Ost, s), 1623 (s), 1449 (s), 1419 (s), 1367 (s), 1266 (s), 1216 (s), 1170 (s), 1082 (s), 1038 (s), 984 (s), 939 (m), 857 (s), 820 (s), 776 (s), 732 (m), $678(\mathrm{w}) \mathrm{cm}^{-1} .{ }^{1} \mathbf{H}$ NMR $\delta 8.26(\mathrm{~d}, J=16.0$, $\left.1 \mathrm{H}, \mathrm{CH}=\mathrm{CHCO}_{2} \mathrm{Et}\right), 6.13\left(\mathrm{~d}, J=16.0,1 \mathrm{H}, \mathrm{CH}=\mathrm{CHCO}_{2} \mathrm{Et}\right), 4.97(\mathrm{~m}, 1 \mathrm{H}, \mathrm{CHCp}), 4.77(\mathrm{~m}$, $1 \mathrm{H}, \mathrm{CHCp}), 4.56$ (t, $J=2.7,1 \mathrm{H}, \mathrm{CHCp}), 4.20$ (q, $\left.J=7.1,2 \mathrm{H}, \mathrm{CH}_{2} \mathrm{CH}_{3}\right), 4.17$ (s, 5H, Cp), $3.82\left(\mathrm{~s}, 3 \mathrm{H}, \mathrm{CH}_{3} \mathrm{O}\right), 1.31\left(\mathrm{t}, J=7.1,3 \mathrm{H}, \mathrm{CH}_{3} \mathrm{CH}_{2}\right) .{ }^{13} \mathbf{C}$ NMR $\delta 171.7(\mathrm{CpC}=\mathrm{O}), 166.8$ $(\mathrm{CH}=\mathrm{CHC}=\mathrm{O}), 143.7\left(\mathrm{CH}=\mathrm{CHCO}_{2} \mathrm{Et}\right), 117.4\left(\mathrm{CH}=\mathrm{CHCO}_{2} \mathrm{Et}\right), 80.3(\mathrm{CCp}), 73.6(\mathrm{CHCp})$, 72.0 (CHCp), $71.4(\mathrm{Cp}), 71.2(\mathrm{CCp}), 69.3(\mathrm{CHCp}), 60.2\left(\mathrm{CH}_{2} \mathrm{CH}_{3}\right), 51.7\left(\mathrm{CH}_{3} \mathrm{O}\right), 14.3$ $\left(\mathrm{CH}_{3} \mathrm{CH}_{2}\right.$ ). MS (EI, $\left.70 \mathrm{eV}\right) \mathrm{m} / z 343$ ([M+1] $]^{+}$, 5), 342 ([M]+., 30), 329 (17), 328 (96), 297 (6), 277 ([M-Cp] $\left.^{+}, 3\right), 263$ (13), 245 (12), 233 (16), 231 (67), 201 (20), 152 (23), 145 (78), 122 (60), 117 (85), 89 (100), 56 ([Fe] $]^{+}$, 45). HRMS (EI, $70 \mathrm{eV}$ ) calcd. for $\mathrm{C}_{17} \mathrm{H}_{18} \mathrm{FeO}_{4} 342.0554$. Found 342.055 .

\section{II.3. Synthesis of 7 through conjugate cuprate addition}

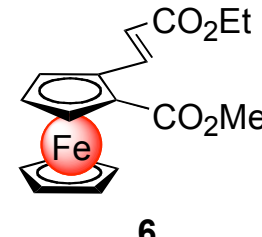

6

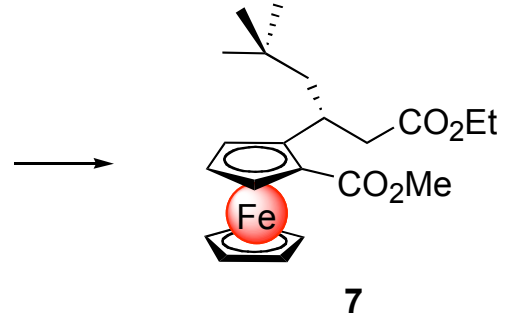

7

To a mixture of magnesium turnings (988 mg, 1.2 equiv., $40.65 \mathrm{mmol})$ in dry THF $(10 \mathrm{~mL})$ was added under argon atmosphere a small iodine crystal. After activation, 1-bromo-2,2dimethylpropane (4.3 mL, 1 equiv., $33.88 \mathrm{mmol})$ in THF (24 mL) was added dropwise so as to maintain a gentle reflux and the mixture was stirred overnight at room temperature. The resulting $1 \mathrm{M}$ solution of 2,2-dimethylpropanemagnesium bromide in THF $(12.5 \mathrm{~mL}, 6$ 
equiv., $12.5 \mathrm{mmol}$ ) was added dropwise at $-78^{\circ} \mathrm{C}$ to a mixture of $\mathrm{CuBr}$. $\mathrm{Me}_{2} \mathrm{~S}(257 \mathrm{mg}, 0.6$ equiv., $1.25 \mathrm{mmol})$ in THF ( $3 \mathrm{~mL})$ under argon atmosphere. The temperature was allowed to warm to $-30^{\circ} \mathrm{C}$ for $10 \mathrm{~min}$ to give a white slurry and was recooled to $-78^{\circ} \mathrm{C}$. $\mathrm{TMSCl}(530 \mu \mathrm{L}$, 2 equiv., $4.15 \mathrm{mmol}$ ) and then the $\alpha, \beta$-unsaturated ferrocenylester 6 (710 mg, 1 equiv., 2.07 mmol) in THF $(9.1 \mathrm{~mL})$ were added dropwise successively. The mixture was stirred for $1 \mathrm{~h}$ at low temperature (below $-60^{\circ} \mathrm{C}$ ) and allowed to warm slowly to $-30^{\circ} \mathrm{C}$, stirred $1 \mathrm{~h}$ at this temperature and finally warm to $0^{\circ} \mathrm{C}$. Saturated aqueous $\mathrm{NH}_{4} \mathrm{Cl}$ solution was added and the product was extracted with MTBE. The organic layer was washed with brine, dried over $\mathrm{Na}_{2} \mathrm{SO}_{4}$, filtered and concentrated under vacuum. Flash chromatography (cyclohexane/ethyl acetate 10/1 then 6/1 and then 3/1) gave 7 as a brown oil (839 mg, $2.03 \mathrm{mmol}, 98 \%$ yield). $[\alpha]^{\mathbf{2 0}}{ }_{\mathbf{D}}-24.5\left(c\right.$ 0.375, $\left.\mathrm{CHCl}_{3}\right)$. IR (neat) v $3095(\mathrm{w}), 2949(\mathrm{~m}), 2901(\mathrm{w}), 1731(\mathrm{C}=\mathrm{Ost}, \mathrm{s})$, 1713 (C=Ost, s), 1451 (m), 1365 (m), 1292 (m), 1210 (m), 1189 (m), 1147 (m), 1085 (m), $1033(\mathrm{~m}), 818(\mathrm{w}), 776(\mathrm{w}) \mathrm{cm}^{-1} .{ }^{1} \mathbf{H}$ NMR $\delta 4.64\left(\mathrm{dd}, J=2.6,1.5,1 \mathrm{H}, \mathrm{CHCH}_{2} \mathrm{CO}_{2} \mathrm{Et}\right), 4.21$ (t, $J=2.6,1 \mathrm{H}, \mathrm{CHCp}), 4.17$ (m, 2H, CHCp), 4.09 (q, $J=7.1,2 \mathrm{H}, \mathrm{CH}_{2} \mathrm{CH}_{3}$ ), 4.04 (s, 5H, Cp), $3.70\left(\mathrm{~s}, 3 \mathrm{H}, \mathrm{CH}_{3} \mathrm{O}\right), 3.04\left(\mathrm{dd}, J=15.8,4.4,1 \mathrm{H}, \mathrm{CHHCO}_{2} \mathrm{Et}\right), 2.60(\mathrm{dd}, J=15.7,8.7,1 \mathrm{H}$, $\left.\mathrm{CH} H \mathrm{CO}_{2} \mathrm{Et}\right), 1.42\left(\mathrm{dd}, J=14.0,5.4,1 \mathrm{H}, \mathrm{CHHC}\left(\mathrm{CH}_{3}\right)_{3}\right), 1.30\left(\mathrm{~m}, 1 \mathrm{H}, \mathrm{CH} H \mathrm{C}\left(\mathrm{CH}_{3}\right)_{3}\right), 1.20$ (t, $\left.J=7.1,3 \mathrm{H}, \mathrm{CH}_{3} \mathrm{CH}_{2}\right), 0.66\left(\mathrm{~s}, 9 \mathrm{H},\left(\mathrm{CH}_{3}\right)_{3} \mathrm{C}\right) .{ }^{13} \mathrm{C}$ NMR $\delta 172.8(\mathrm{C}=\mathrm{O}), 172.3(\mathrm{C}=\mathrm{O}), 99.9$ $(C \mathrm{CpC}=\mathrm{O}), 70.6(\mathrm{CHCp}), 70.1(\mathrm{Cp}), 69.4(\mathrm{CHCp}), 69.1(\mathrm{CHCp}), 68.5(\mathrm{CCpCH}), 60.3$

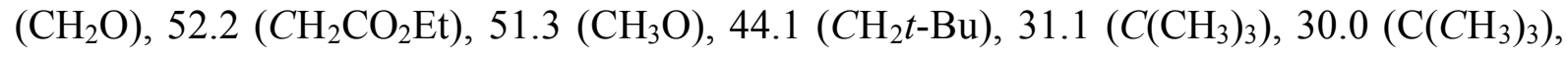
$29.1\left(\mathrm{CHCH}_{2} \mathrm{CO}_{2} \mathrm{Et}\right), 14.3\left(\mathrm{CH}_{3} \mathrm{CH}_{2}\right)$. MS (EI, $\left.70 \mathrm{eV}\right) \mathrm{m} / \mathrm{z}=415\left([\mathrm{M}+1]^{+}, 25\right), 414\left([\mathrm{M}]^{+}\right.$, 100), 343 ([M-( $\left.\left(\mathrm{CH}_{2} t-\mathrm{Bu}\right)\right]^{+}$, 2), 297 (8), 257 (11), 239 (8), 175 (13), 121 ([FeCp] , 22), 105 (14), 91 (14), 57 (32). HRMS (EI, $70 \mathrm{eV}$ ) calcd. for $\mathrm{C}_{22} \mathrm{H}_{30} \mathrm{FeO}_{4}$ 414.1493. Found 414.149.

\section{II.4. Synthesis of ketone 8 by Dieckmann cyclization}

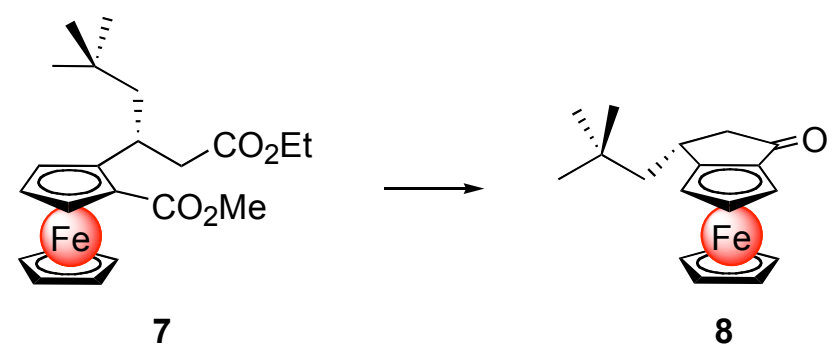

General procedure: To a suspension of $\mathrm{NaH}(60 \%$ in mineral oil, washed with dry hexane, 4 equiv.) in THF (2.5 mL/mmol) under argon atmosphere was added the diester (1 equiv.) in THF $(25 \mathrm{~mL} / \mathrm{mmol})$ at room temperature. The orange mixture was then stirred under reflux for $7 \mathrm{~h}$. The obtained dark red solution was cooled in an ice bath, $\mathrm{MeOH}$ was added slowly, 
and then MTBE. The organic layer was washed with a $1 \mathrm{M}$ aqueous solution of $\mathrm{HCl}$ then with brine, dried over $\mathrm{Na}_{2} \mathrm{SO}_{4}$, filtered and concentrated under vacuum. The crude product was directly used for the next step without further purification.

Following the general procedure described above, the diester $7(832 \mathrm{mg}, 2.01 \mathrm{mmol})$ and $\mathrm{NaH}$ (321 mg, $8.03 \mathrm{mmol})$ were reacted. The crude product $(2.01 \mathrm{mmol})$ was dissolved in ethanol $(20 \mathrm{~mL})$ and a $1 \mathrm{M}$ solution of $\mathrm{NaOH}(20 \mathrm{~mL})$ was added. The mixture was heated at $+80^{\circ} \mathrm{C}$ and stirred overnight at this temperature. After cooling to room temperature, MTBE was added and the layers were separated. The aqueous layer was extracted with MTBE and the organic layers were combined. After washing with brine, the solution was dried over $\mathrm{Na}_{2} \mathrm{SO}_{4}$, filtered, and concentrated under vacuum. Purification by flash chromatography (cyclohexane/ethyl acetate 3/1) afforded 8 as a red oil which then solidified (393 $\mathrm{mg}, 63 \%$ yield, 2 steps). $\mathbf{M p :}+82.2-82.8^{\circ} \mathrm{C} .[\alpha]^{\mathbf{2 0}}{ }_{\mathbf{D}}+213.6\left(c\right.$ 0.355, $\left.\mathrm{CHCl}_{3}\right)$. IR (neat) $v 3095(\mathrm{w})$, 2950 (m), 2906 (w), 2863 (w), 1699 (C=Ost, s), 1461 (m), 1363 (m), 1291 (m), 1196 (w), $1106(\mathrm{~m}), 1073(\mathrm{w}), 1001(\mathrm{w}), 821(\mathrm{~m}) \mathrm{cm}^{-1} .{ }^{1} \mathbf{H}$ NMR $\delta 4.58(\mathrm{~m}, 1 \mathrm{H}, \mathrm{CpH}), 4.49(\mathrm{~m}, 1 \mathrm{H}$, $\mathrm{CpH}), 4.38(\mathrm{~m}, 1 \mathrm{H}, \mathrm{CpH}), 4.18(\mathrm{~s}, 5 \mathrm{H}, \mathrm{Cp}), 3.00-2.80\left(\mathrm{~m}, 3 \mathrm{H}, \mathrm{CHCH} \mathrm{C}_{2} \mathrm{C}=\mathrm{O}\right), 1.93$ (dd, $J=$ 13.7, 2.4, 1H, CHHt-Bu), $1.60(\mathrm{dd}, J=14.0,8.5,1 \mathrm{H}, \mathrm{CHH} t-\mathrm{Bu}), 0.95$ (s, 9H, $t-\mathrm{Bu}) .{ }^{13} \mathrm{C}$ NMR $\delta 207.2(\mathrm{C}=\mathrm{O}), 111.3(\mathrm{CCpC}=\mathrm{O}), 79.8(\mathrm{CCp}), 75.2(\mathrm{CHCp}), 70.1(\mathrm{Cp}), 65.2$ (CHCp),

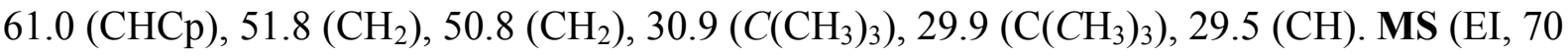
eV) $\left.m / z 311\left([\mathrm{M}+1]^{+}, 22\right), 310\left([\mathrm{M}]^{+}, \text {, 100), } 282 \text { ([M-(CO) }\right]^{+}, 3\right), 267$ ([M-(CO)- $\left.\left.\left(\mathrm{CH}_{3}\right)\right]^{+}, 5\right)$, $253\left([\mathrm{M}-(t-\mathrm{Bu})]^{+},, 9\right), 239\left(\left[\left(\mathrm{M}-(t-\mathrm{Bu})-\left(\mathrm{CH}_{2}\right)\right]^{+}, 35\right), 226(21), 225\left([\mathrm{M}-(\mathrm{CO})-(t-\mathrm{Bu})]^{+}, 43\right)\right.$, 211 ([M-(CO)-( $\left.\left.\mathrm{CH}_{2} t-\mathrm{Bu}\right)\right]^{+}$, 22), $186\left(\left[\mathrm{FeCp}_{2}\right]^{+}, 6\right), 153$ (17), 133 (17), 121 ([FeCp] $\left.{ }^{+}, 58\right), 56$ $\left([\mathrm{Fe}]^{+}\right.$, , 32). HRMS (EI, $70 \mathrm{eV}$ ) calcd. for $\mathrm{C}_{18} \mathrm{H}_{22} \mathrm{FeO} 310.1020$. Found 310.102. Elem. Anal. calcd. for $\mathrm{C}_{18} \mathrm{H}_{22} \mathrm{FeO} \mathrm{C} 69.69, \mathrm{H}$ 7.15. Found C 69.54, H 7.11.

\section{II.5. Synthesis of 9 through reduction and acetylation}

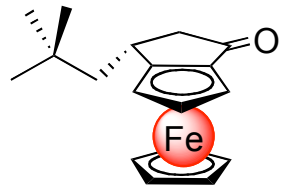

8

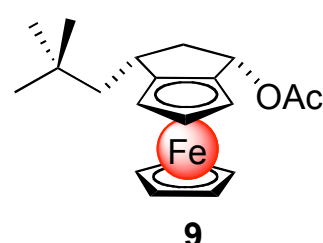

9

General Procedure: The $\alpha$-ferrocenylketone (1 equiv.) was dissolved in a 4:1 mixture of ethanol:dioxane. $\mathrm{NaBH}_{4}$ (5 equiv.) was then added slowly in portions at room temperature. Conversion into the corresponding alcohol was easily observed by the change of the color of the solution, from red to yellow. An aqueous solution of $\mathrm{NH}_{4} \mathrm{Cl}$ was then added at $0^{\circ} \mathrm{C}$ and the alcohol was extracted with MTBE. The organic layer was washed with brine, dried over 
$\mathrm{Na}_{2} \mathrm{SO}_{4}$, filtered and concentrated under vacuum. Crude products were pure enough to be directly used for the next step without further purification. The obtained $\alpha$-ferrocenylalcohol (1 equiv.) was dissolved in dry pyridine $(6 \mathrm{~mL} / \mathrm{mmol})$ under argon atmosphere and acetic anhydride $(2.3 \mathrm{~mL} / \mathrm{mmol})$ was added dropwise. After stirring overnight at room temperature, a saturated aqueous solution of $\mathrm{NH}_{4} \mathrm{Cl}$ was added. The acetate was extracted with $\mathrm{CH}_{2} \mathrm{Cl}_{2}$ and the organic layer was washed successively with saturated aqueous $\mathrm{NaHCO}_{3}$ and brine. The organic solution was then dried with $\mathrm{Na}_{2} \mathrm{SO}_{4}$, filtered and concentrated under vacuum. Purification by flash chromatography afforded the corresponding acetate.

Following the general procedure described above, ketone $\mathbf{8}$ was converted into the acetate $\mathbf{9}$ using the following amounts of starting materials and reagents: ketone 8 (105 mg, 1 equiv., $0.34 \mathrm{mmol}), \mathrm{MeOH}$ :dioxane (2 mL:500 $\mu \mathrm{L}), \mathrm{NaBH}_{4}$ (64 mg, 5 equiv., $\left.1.70 \mathrm{mmol}\right)$. After 30 min and classical work-up, the alcohol (96 mg, d.r $\geq 92: 8$ ) was used without further purification. It was then reacted in pyridine $(2 \mathrm{~mL})$ with acetic anhydride $(780 \mu \mathrm{L}), 5 \mathrm{~h}$, rt. Purification by flash chromatography (cyclohexane/ethyl acetate 9/1) afforded $\mathbf{9}$ as a yellow oil (110 mg, $0.31 \mathrm{mmol}, 90 \%$ over 2 steps). $[\alpha]^{20}{ }_{\mathbf{D}}-117.5,[\alpha]_{365}^{20}-257.3,[\alpha]^{20}{ }_{546}-148.1(c$ 0.34, $\mathrm{CHCl}_{3}$ ). IR (neat) v $3093(\mathrm{w}), 2952$ (m), 2865 (w), 1737 (C=Ost, s), 1473 (w), 1365 (m), 1237 (s), 1105 (m), 1037 (m), $816(\mathrm{~m}) \mathrm{cm}^{-1} .{ }^{1}$ H NMR $\delta 5.44$ (t, $J=7.5,1 \mathrm{H}$, CHOAc), 4.27 (s, 5H, Cp), 4.13 (m, 1H, CHCp), 4.02 (m, 1H, CHCp), 3.92 (m, 1H, CHCp), 2.85 (m, 1H, CHHCHOAc), $2.43\left(\mathrm{~m}, 1 \mathrm{H}, \mathrm{CHCH}_{2} t-\mathrm{Bu}\right), 2.19\left(\mathrm{~s}, 3 \mathrm{H}, \mathrm{CH}_{3} \mathrm{CO}\right), 2.10(\mathrm{~m}, 1 \mathrm{H}$, CHHCHOAc), 1.88 (dd, $J=14.0,3.3,1 \mathrm{H}, \mathrm{CHH} t-\mathrm{Bu}), 1.58$ (dd, $J=14.0,7.6,1 \mathrm{H}, \mathrm{CH} H t-\mathrm{Bu}$ ), $1.00(\mathrm{~s}, 9 \mathrm{H}, t-\mathrm{Bu}) .{ }^{13} \mathrm{C}$ NMR $\delta 201.4(\mathrm{C}=\mathrm{O}), 102.6$ (CCp), 92.2 (CCp), 73.0 (CHOAc), 69.9 (CHCp), 68.6 (Cp), 60.3 (CHCp), 59.9 (CHCp), $50.1\left(\mathrm{CH}_{2} t-\mathrm{Bu}\right), 45.0\left(\mathrm{CH}_{2} \mathrm{CHOAc}\right), 31.5$ $\left(\mathrm{CHCH}_{2} t-\mathrm{Bu}\right), 30.8\left(C\left(\mathrm{CH}_{3}\right)_{3}\right), 30.0\left(\left(\mathrm{CH}_{3}\right)_{3} \mathrm{C}\right), 21.1\left(\mathrm{CH}_{3} \mathrm{CO}\right)$. MS (EI, $\left.70 \mathrm{eV}\right) \mathrm{m} / z 355$ $\left([\mathrm{M}+1]^{+}, 5\right), 354\left([\mathrm{M}]^{+}, 25\right), 294$ (26), 237 (43), $180(100), 121$ ([FeCp $\left.]^{+}, 51\right), 103$ (26), 57 (30). HRMS (EI, $70 \mathrm{eV}$ ) calcd. for $\mathrm{C}_{20} \mathrm{H}_{26} \mathrm{FeO}_{2}$ 354.1282. Found 354.129.

\section{II.6. General procedure for the synthesis of nucleosides using silylated nucleobases:}

To a solution of the $\alpha$-ferrocenylacetate (1 equiv.) and TMS-protected nucleobase (4 equiv.) in dry $\mathrm{CH}_{2} \mathrm{Cl}_{2}$ under argon atmosphere was added dropwise at $0^{\circ} \mathrm{C}$ TMSOTf (6 equiv.). The yellow solution was stirred in an ice bath for $0.5-1.5 \mathrm{~h}$ and then treated with a saturated solution of $\mathrm{NaHCO}_{3}$. The phases were separated and the aqueous phase washed with $\mathrm{CH}_{2} \mathrm{Cl}_{2}$. The combined organic layers were then washed with brine, dried with $\mathrm{Na}_{2} \mathrm{SO}_{4}$, filtered and the solvent removed under vacuum. Purification was achieved by flash chromatography. 


\section{II.7. Synthesis of the cytosine derivative 10 .}

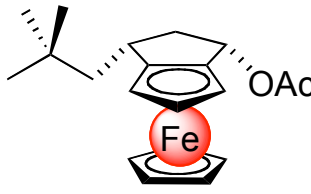

9

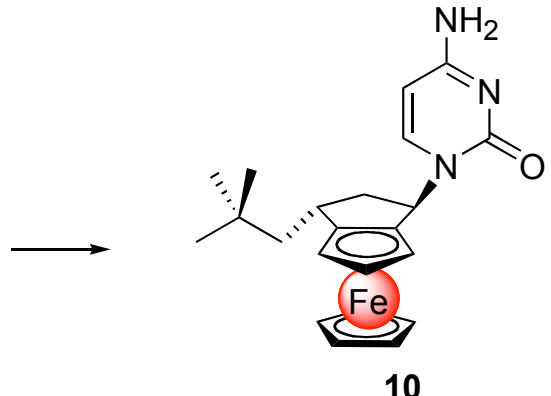

10

The general procedure for nucleobase introduction was used (see II.6.). Amounts: acetate 9 (194 mg, 1 equiv., $0.63 \mathrm{mmol}$ ), (TMS) $)_{2}$ cytosine (643 mg, 4 equiv., $2.50 \mathrm{mmol}$ ), in $\mathrm{CH}_{2} \mathrm{Cl}_{2}$ (14 $\mathrm{mL}$ ), TMSOTf (685 $\mu \mathrm{L}, 6$ equiv., $3.78 \mathrm{mmol}$ ), stirred for $1.5 \mathrm{~h}$. Flash chromatography (ethyl acetate/methanol 95/5) afforded $\mathbf{1 0}$ as a yellow powder (205 $\mathrm{mg}, 0.51 \mathrm{mmol}, 80 \%$ yield). Mp: decomposition starts at $\mathrm{T}>200^{\circ} \mathrm{C} .[\alpha]^{\mathbf{2 0}}{ }_{\mathrm{D}}-203\left(c 0.29, \mathrm{CHCl}_{3}\right)$. IR (neat) $v 3345$ (NHst, $\mathrm{m}$ ), 3201 (NHst, m), 3092 (w), 2949 (m), 2860 (w), 1643 (C=Ost, s), 1522 (m), 1484 (s), 1395 (m), 1275 (m), 1105 (w), 1030 (w), 999 (w), 788 (m), 751 (w) $\mathrm{cm}^{-1} .{ }^{1}$ H NMR $\delta 6.73$ (d, $J=$ 7.0, 1H, NCH=CHC), 5.67 (d, $J=6.2,1 \mathrm{H}, \mathrm{NCH}=\mathrm{CHC}), 5.60$ (d, $J=6.6,1 \mathrm{H}$, CHcytosine), 4.20 (m, 1H, CHCp), 4.17 (s, 5H, Cp), 4.85 (m, 1H, CHCp), 4.04 (m, 1H, CHCp), 2.61 (m, 1H, CHHCHcytosine), 2.40 (m, 2H, CHCHHCHcytosine), 1.80 (d, $J=13.7,1 \mathrm{H}, \mathrm{CHH}$-Bu), $1.45(\mathrm{dd}, J=13.4,6.3,1 \mathrm{H}, \mathrm{CH} H t-\mathrm{Bu}), 0.91$ (s, 9H, $t$-Bu). MS (EI, $70 \mathrm{eV}) \mathrm{m} / z 406\left([\mathrm{M}+1]^{+}\right.$, 2), $405\left([\mathrm{M}]^{+}, 7\right), 295$ ([M-(cytosine) $\left.]^{+}, 22\right), 294$ (100), 238 ([M-(cytosine)-( $t$-Bu) $\left.]^{+}, 19\right), 237$ (62), 232 (13), 231 (51), 172 (11), 137 (21), 121 ([FeCp] $\left.]^{+}, 52\right), 103$ (18), 69 (12), 57 (16), 56 ([Fe $\left.]^{+}, 19\right)$. HRMS (EI, $70 \mathrm{eV}$ ) calcd. for $\mathrm{C}_{22} \mathrm{H}_{27} \mathrm{FeN}_{3} \mathrm{O}$ 405.1503. Found 405.150.

\section{II.8. Synthesis of the uracil derivative 11.}
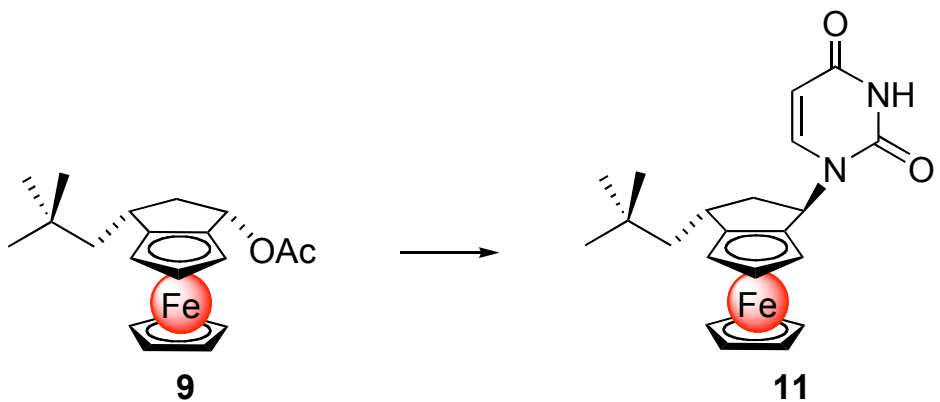

The general procedure for nucleobase introduction was used (see II.6.). Amounts: acetate 9 (64 mg, $0.21 \mathrm{mmol}),(\mathrm{TMS})_{2}$ uracil (213 mg, $\left.0.83 \mathrm{mmol}\right)$, in $\mathrm{CH}_{2} \mathrm{Cl}_{2}$ (4.7 mL), TMSOTf (230 $\mu \mathrm{L}, 1.26 \mathrm{mmol}$ ), stirred for $0.5 \mathrm{~h}$. Flash chromatography (ethyl acetate/methanol 1/1) afforded 11 as a yellow-orange powder (61 mg, $0.15 \mathrm{mmol}, 72 \%$ yield). Mp: decomposition starts when $\mathrm{T}>220^{\circ} \mathrm{C} .[\alpha]^{\mathbf{2 0}}{ }_{\mathrm{D}}-179,[\alpha]^{20}{ }_{546}-242\left(c 0.38, \mathrm{CHCl}_{3}\right)$. IR (neat) $v 3263$ (NHst, w), 3094 
(w), 2951 (w), 2863 (w), 1692 (C=Ost, s), 1680 (C=Ost, s), 1462 (w), 1377 (w), $1364(\mathrm{w})$, 1258 (m), 1213 (w), 1175 (w), 1105 (w), 1000 (w), 815 (w), 765 (w) cm ${ }^{-1} .{ }^{1}$ H NMR $\delta 9.40$ (br s, 1H, NH), 6.65 (d, $J=8.0,1 \mathrm{H}, \mathrm{NCH}=\mathrm{CHCO}), 5.63,(\mathrm{~d}, J=6.5,1 \mathrm{H}, \mathrm{CHN}), 5.49(\mathrm{~d}, J=$ 8.0, 1H, $\mathrm{NCH}=\mathrm{CHCO}$ ), 4.23 (br s, 1H, CHCp), 4.19 (s, 5H, Cp), 4.13 (br s, 1H, CHCp), 4.07 (br s, $1 \mathrm{H}, \mathrm{CHCp}), 2.70(\mathrm{~m}, 1 \mathrm{H}, \mathrm{CH} H \mathrm{CHN}), 2.51\left(\mathrm{~m}, 1 \mathrm{H}, \mathrm{CHCH}_{2} t-\mathrm{Bu}\right), 2.43(\mathrm{~m}, 1 \mathrm{H}$, $\mathrm{CHHCHN}), 1.83(\mathrm{dd}, J=13.9,2.5,1 \mathrm{H}, \mathrm{CHH} t-\mathrm{Bu}), 1.50(\mathrm{dd}, J=14.0,7.1,1 \mathrm{H}, \mathrm{CH} H \mathrm{t}-\mathrm{Bu})$, $0.95(\mathrm{~s}, 9 \mathrm{H}, t-\mathrm{Bu}) .{ }^{13} \mathrm{C}$ NMR $\delta 163.5(\mathrm{C}=\mathrm{O}), 151.0(\mathrm{NHC}=\mathrm{ON}), 140.9(\mathrm{NCH}=\mathrm{CHCO}), 103.3$ (CCp), 101.5 (NCH=CHCO), 86.9 (CCp), 71.5 (CHCp), 69.4 (Cp), 61.9 (CHCp), 61.7

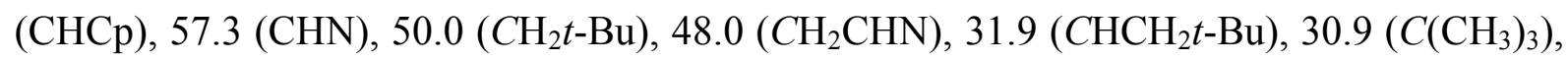
$30.1\left(\mathrm{CH}_{3}\right)$. MS (EI, $\left.70 \mathrm{eV}\right) \mathrm{m} / z 407$ ([M+1] $\left.]^{+}, 13\right), 406$ ([M] ${ }^{+}$, , 47), 295 ([M-(uracil) $]^{+}$, 18),

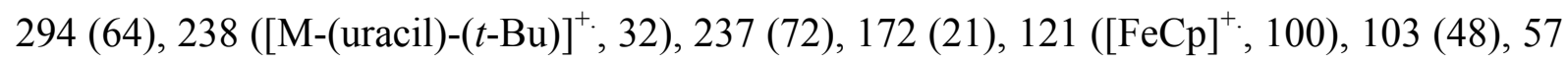
(16). HRMS (EI, $70 \mathrm{eV}$ ) calcd. for $\mathrm{C}_{22} \mathrm{H}_{26} \mathrm{FeN}_{2} \mathrm{O}_{2}$ 406.1343. Found 406.134.

\section{II.9. Synthesis of 13 and 14a through Mukaiyama-Michael addition.}

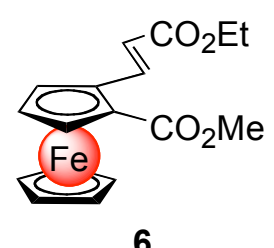

6
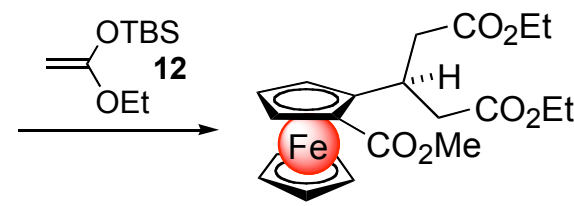

13

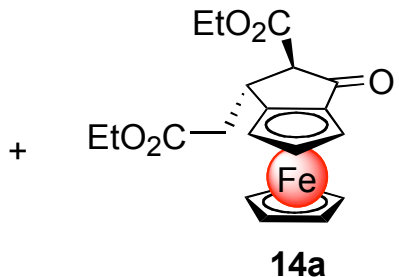

$14 a$

To a solution of trifluoromethanesulfonic acid (150 $\mu \mathrm{L}, 0.57$ equiv., $1.66 \mathrm{mmol})$ in $\mathrm{CH}_{2} \mathrm{Cl}_{2}$ $(15.3 \mathrm{~mL})$ under argon atmosphere was added dropwise at $-78^{\circ} \mathrm{C}$ a $2 \mathrm{M}$ solution of $\mathrm{AlMe}_{3}$ in toluene $(290 \mu \mathrm{L}, 0.20$ equiv., $0.58 \mathrm{mmol})$. After $5 \mathrm{~min}$ at this temperature, the mixture was stirred $30 \mathrm{~min}$ at room temperature. The mixture was then recooled to $-78^{\circ} \mathrm{C}$ and the ester 6 ( $1 \mathrm{~g}$, 1 equiv., $2.92 \mathrm{mmol}$ ) in $\mathrm{CH}_{2} \mathrm{Cl}_{2}(9.5 \mathrm{~mL}$ ) was added dropwise, followed by the addition of the silylenolether $\mathbf{1 2}^{3}$ (930 mg, 1.57 equiv., $4.58 \mathrm{mmol}$ ) in $\mathrm{CH}_{2} \mathrm{Cl}_{2}(9.5 \mathrm{~mL}$ ). The solution was stirred for $2.5 \mathrm{~h}$ at $-78^{\circ} \mathrm{C}^{4}$ and $\mathrm{MeOH}$ was then added. After warming to $0^{\circ} \mathrm{C}$, saturated $\mathrm{NH}_{4} \mathrm{Cl}$ was added and the layers were separated. The aqueous layer was washed with $\mathrm{CH}_{2} \mathrm{Cl}_{2}$ and the combined organic layers were washed with brine, dried over $\mathrm{MgSO}_{4}$, filtered and concentrated under vacuum. Purification by flash chromatography (cyclohexane/ethyl acetate $3 / 1)$ afforded the addition product $\mathbf{1 3}$ as an orange oil $(635 \mathrm{mg}, 58 \%)$ and the cyclized product 14a as a dark red oil (397 mg, 34\%, 92\% global yield). ${ }^{4}$

\footnotetext{
${ }^{3}$ Kita, Y.; Segawa, J.; Haruta, J.-i.; Yasuda, H.; Tamura, Y. J. Chem. Soc., Prekin Trans. 1 1982, 1099.

${ }^{4}$ When the temperature was allowed to warm slowly to room temperature after $30 \mathrm{~min}$. and stirred overnight at r.t., $43 \%$ of 14 a was isolated as a single diastereomer besides $47 \%$ of 13 (90\% global yield).
} 
Data for addition product 13:

$[\alpha]^{\mathbf{2 0}}{ }_{\mathbf{D}}-42.6,[\alpha]^{20}{ }_{546}-52.4\left(c 0.385, \mathrm{CHCl}_{3}\right)$. IR (neat) $v 3092(\mathrm{w}), 2977(\mathrm{~m}), 2948(\mathrm{~m}), 1731$ (C=Ost, s), 1711 (C=Ost, s), 1445 (m), 1370 (m), 1340 (w), 1293 (m), 1216 (m), 1158 (s), $1090(\mathrm{~m}), 818(\mathrm{w}) \mathrm{cm}^{-1} .{ }^{1} \mathbf{H}$ NMR $\delta 4.65$ (s, 1H, CHCp), 4.16 (s, $\left.2 \mathrm{H}, \mathrm{CHCp}\right), 4.05$ (q, $J=$ 7.1, 2H, $\left.\mathrm{CH}_{2} \mathrm{CH}_{3}\right), 4.02(\mathrm{~s}, 5 \mathrm{H}, \mathrm{Cp}), 3.90\left(\mathrm{~m}, 1 \mathrm{H}, \mathrm{CH}\left(\mathrm{CH}_{2} \mathrm{CO}_{2} \mathrm{Et}\right)_{2}\right), 3.80$ (q, $J=7.1$, $\left.\mathrm{CH}_{2} \mathrm{CH}_{3}\right), 3.66\left(\mathrm{~s}, 3 \mathrm{H}, \mathrm{CH}_{3} \mathrm{O}\right), 2.90\left(\mathrm{dd}, J=15.4,4.1,1 \mathrm{H}, \mathrm{CHCHHCO}_{2} \mathrm{Et}\right), 2.78$ (dd, $J=$ 15.3, 9.7, $\left.1 \mathrm{H}, \mathrm{CHCH} H C O_{2} \mathrm{Et}\right), 2.43\left(\mathrm{~m}, 2 \mathrm{H}, \mathrm{CHCH}_{2} \mathrm{CO}_{2} \mathrm{Et}\right), 1.17$ (t, $J=7.1,3 \mathrm{H}, \mathrm{CH}_{3} \mathrm{CH}_{2}$ ), $0.99\left(\mathrm{t}, J=7.1,3 \mathrm{H}, \mathrm{CH}_{3} \mathrm{CH}_{2}\right) .{ }^{13} \mathbf{C}$ NMR $\delta 172.0(\mathrm{C}=\mathrm{O}), 171.8(\mathrm{C}=\mathrm{O}), 171.3(\mathrm{C}=\mathrm{O}), 93.5$ (CCp), 70.5 (CHCp), 70.3 (CHCp), 69.9 (Cp), 69.0 (CHCp), 68.0 (CCp), $60.1\left(\mathrm{CH}_{2} \mathrm{O}\right), 59.6$ $\left(\mathrm{CH}_{2} \mathrm{O}\right), 51.0\left(\mathrm{CH}_{3} \mathrm{O}\right), 39.9\left(\mathrm{CH}_{2} \mathrm{CO}_{2} \mathrm{Et}\right), 37.9\left(\mathrm{CH}_{2} \mathrm{CO}_{2} \mathrm{Et}\right), 30.8\left(\mathrm{CH}\left(\mathrm{CH}_{2} \mathrm{CO}_{2} \mathrm{Et}\right)_{2}\right), 13.9$ $\left(\mathrm{CH}_{3} \mathrm{CH}_{2}\right), 13.8\left(\mathrm{CH}_{3} \mathrm{CH}_{2}\right)$. MS (EI, $\left.70 \mathrm{eV}\right) \mathrm{m} / z 431\left([\mathrm{M}+1]^{+}, 25\right), 430\left([\mathrm{M}]^{+}, 100\right), 319(17)$, 121 ([FeCp $]^{+}$, 24). HRMS (EI, $70 \mathrm{eV}$ ) calcd. for $\mathrm{C}_{21} \mathrm{H}_{26} \mathrm{FeO}_{6} 430.1078$. Found 430.108.

Data for cyclization product $\mathbf{1 4 a}$ :

$[\alpha]^{\mathbf{2 0}}{ }_{\mathbf{D}}+309\left(c\right.$ 0.085, $\left.\mathrm{CHCl}_{3}\right)$. IR (neat) v $3105(\mathrm{w}), 2978(\mathrm{w}), 2928(\mathrm{w}), 1731(\mathrm{C}=\mathrm{Ost}, \mathrm{s})$, 1703 (C=Ost, s), 1462 (w), 1425 (w), 1369 (w), 1327 (w), 1255 (m), 1154 (m), 1106 (w), $1026(\mathrm{~m}), 822(\mathrm{w}) \mathrm{cm}^{-1} .{ }^{1} \mathbf{H}$ NMR $\delta 4.64$ (br s, 1H, CHCp), 4.55 (br s, 1H, CHCp), 4.41 (br s, 1H, CHCp), 4.19 (s, 5H, Cp), 4.15 (m, 4H, $\mathrm{CH}_{2} \mathrm{CH}_{3}$ ), 3.73 (d, J=6.2, 1H, CHCO), 3.66 (m, $1 \mathrm{H}, \mathrm{CHCHCO}), 2.90(\mathrm{dd}, J=15.6,6.9,1 \mathrm{H}, \mathrm{CH} \mathrm{HCHCHCO}), 2.82(\mathrm{dd}, J=15.8,7.7,1 \mathrm{H}$, $\mathrm{CH} H \mathrm{CHCHCO}), 1.23\left(\mathrm{~m}, 6 \mathrm{H}, \mathrm{CH}_{3} \mathrm{CH}_{2}\right) .{ }^{13} \mathbf{C}$ NMR $\delta 199.0(\mathrm{C}=\mathrm{O}), 171.4(\mathrm{C}=\mathrm{O}), 168.9$ $(\mathrm{C}=\mathrm{O}), 106.5(\mathrm{CCp}), 78.9$ (CCp), 76.3 (CHCp), 70.5 (Cp), $66.2(\mathrm{CHCp}), 65.1\left(\mathrm{CHCO}_{2} \mathrm{Et}\right)$, 61.9 (CHCp), $61.5\left(\mathrm{CH}_{2} \mathrm{CH}_{3}\right), 60.9\left(\mathrm{CH}_{2} \mathrm{CH}_{3}\right), 40.3\left(\mathrm{CH}_{2} \mathrm{CO}_{2} \mathrm{Et}\right), 34.0\left(\mathrm{CHCH}_{2} \mathrm{CO}_{2} \mathrm{Et}\right), 14.2$ $\left(\mathrm{CH}_{3} \mathrm{CH}_{2}\right)$. MS (EI, $\left.70 \mathrm{eV}\right) \mathrm{m} / z 399$ ([M+1] $\left.]^{+}, 15\right), 398\left([\mathrm{M}]^{+}, 68\right), 353$ (21), 352 (45), 325

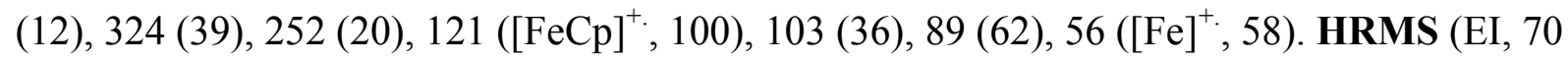
eV) calcd. for $\mathrm{C}_{20} \mathrm{H}_{22} \mathrm{FeO}_{5}$ 398.0816. Found 398.082.

\section{II.10. Synthesis of 14a and 14b through group-selective Dieckmann cyclization.}

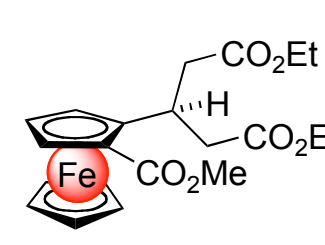

13

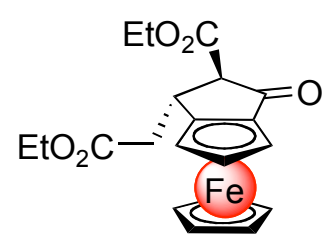

$14 \mathrm{a}$

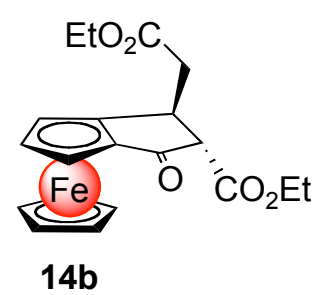

$14 b$ 
Procedure A using LDA: To a solution of triester 13 (1.3 g, 1 equiv., $3.03 \mathrm{mmol}$ ) in THF (10 $\mathrm{mL}$ ) under argon atmosphere was added dropwise at $-78^{\circ} \mathrm{C}$ a solution of LDA in THF (prepared by a dropwise addition at $-78^{\circ} \mathrm{C}$ of a $1.6 \mathrm{M}$ solution of $n$-BuLi (2.1 mL, 1.1 equiv., $3.33 \mathrm{mmol})$ onto DIPA (515 $\mu \mathrm{L}, 1.2$ equiv., $3.64 \mathrm{mmol})$ in THF (33 mL). The solution was then allowed to warm to $0^{\circ} \mathrm{C}$ for $15 \mathrm{~min}$ and used directly.). After the addition, the temperature was allowed to warm slowly to $-60^{\circ} \mathrm{C}$ and was stirred overnight at this temperature. The reaction mixture was quenched at $0^{\circ} \mathrm{C}$ in an ice bath with a saturated aqueous solution of $\mathrm{NH}_{4} \mathrm{Cl}$. The two phases were separated and the aqueous layer was washed with MTBE. The combined organic layers were then washed with brine, dried over $\mathrm{Na}_{2} \mathrm{SO}_{4}$, filtered and the solvents were removed under vacuum. Purification by flash chromatography (cyclohexane/ethyl acetate 3/1) gave a 1:1 mixture of the two cyclized diastereoisomers 14a and $\mathbf{1 4 b}$ as a red oils (1.18 g, $2.97 \mathrm{mmol}, 98 \%)$.

Procedure B using KH: To a mixture of KH (30\% in oil, washed with dry hexane, $770 \mathrm{mg}, 4$ equiv., $5.75 \mathrm{mmol})$ in THF $(10 \mathrm{~mL})$ under argon atmosphere was added the diester $\mathbf{1 3}$ (620 mg, 1 equiv., $1.44 \mathrm{mmol})$ in THF (36 mL). The reaction mixture was refluxed for $1 \mathrm{~h}$. The red solution was then cooled to $0^{\circ} \mathrm{C}$ and water was added slowly and then a $1.0 \mathrm{M}$ solution of aqueous $\mathrm{HCl}$. The layers were separated and the aqueous layer was washed with MTBE. The combined organic layers was washed with $\mathrm{NaHCO}_{3}$, brine, dried over $\mathrm{Na}_{2} \mathrm{SO}_{4}$, filtered and concentrated under vacuum. Purification by flash chromatography (cyclohexane/ethyl acetate 3/1) gave a 89:11 mixture of the two cyclized diastereoisomers 14a and 14b (462 mg, 1.16 mmol, $81 \%)$.

\section{II.11. Synthesis of diol 15 from 14a.}

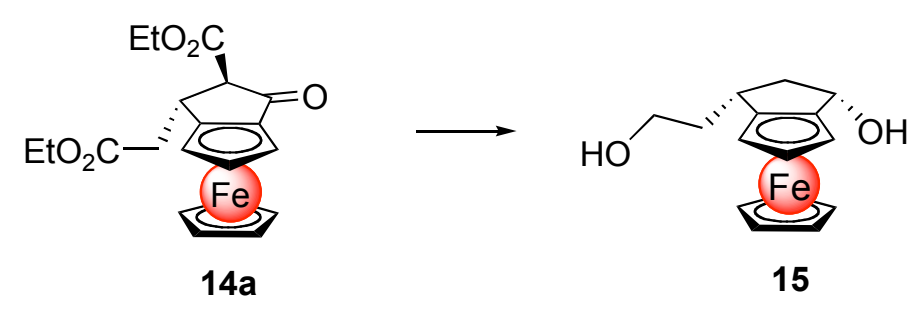

Under argon atmosphere, the diester 14a $(257 \mathrm{mg}, 0.65 \mathrm{mmol})$ was dissolved in ethanol (10 $\mathrm{mL})$ and to this solution was added at room temperature a $1 \mathrm{M}$ aqueous solution of $\mathrm{NaOH}(10$ $\mathrm{mL}$ ). The mixture was then heated at $+80^{\circ} \mathrm{C}$ overnight. After cooling, MTBE was added and the phases separated. The organic layer was washed with a $1.0 \mathrm{M}$ solution of $\mathrm{NaOH}(3 \mathrm{x})$. The combined aqueous layers were then acidified with a $2.0 \mathrm{M}$ solution of $\mathrm{HCl}$ and the carboxylic 
acid extracted with MTBE (3x). The combined MTBE extracts were finally washed with brine, dried over $\mathrm{Na}_{2} \mathrm{SO}_{4}$, filtered and concentrated in vacuo to afford the intermediate monocarboxylic acid. MS (EI, $70 \mathrm{eV}) \mathrm{m} / z 299$ ([M+1] $\left.]^{+}, 15\right), 298\left([\mathrm{M}]^{+}, 100\right), 121$ ([FeCp $\left.]^{+}, 23\right)$, $56\left([\mathrm{Fe}]^{+}\right.$, 25). HRMS (EI, $\left.70 \mathrm{eV}\right)$ calcd. for $\mathrm{C}_{15} \mathrm{H}_{14} \mathrm{FeO}_{3}$ 298.0292. Found 298.029. The crude carboxylic acid (173 mg, 1 equiv., $0.58 \mathrm{mmol})$ was dissolved in THF ( $5 \mathrm{~mL})$ under argon atmosphere and the solution was cooled at $0^{\circ} \mathrm{C}$. A $1 \mathrm{M}$ solution of $\mathrm{LiAlH}_{4}$ in ether $(1.74$ $\mathrm{mL}, 3$ equiv., $1.74 \mathrm{mmol}$ ) was added dropwise. Temperature was allowed to warm slowly to room temperature and the mixture was stirred overnight at room temperature. An aqueous solution of $\mathrm{NaOH} 1 \mathrm{~N}$ was then added carefully. The mixture was then filtered through a pad of celite, rinced many times with $\mathrm{CH}_{2} \mathrm{Cl}_{2}$ and the filtrate was concentrated under vacuum. Purification by flash chromatography (cyclohexane/ethyl acetate 1/1) afforded a yellow oil which solidifies (68 mg, $0.24 \mathrm{mmol}, 41 \%$ for 2 steps). An analytical sample of 15 could be prepared by recristallization in 1,2-dichloroethane. $\mathbf{M p}\left(\mathrm{ClCH}_{2} \mathrm{CH}_{2} \mathrm{Cl}\right):+115-117^{\circ} \mathrm{C} .[\mathbf{\alpha}]^{\mathbf{2 0}} \mathbf{D}$ $+16.5,[\alpha]^{20}{ }_{546}+21.6,\left(c 0.43, \mathrm{CHCl}_{3}\right)$. IR (neat) v 3327 (OHst, m), $3090(\mathrm{w}), 2922(\mathrm{~m}), 2851$ (m), $1725(\mathrm{w}), 1673$ (w), 1447 (m), 1410 (m), 1327 (m), 1260 (s), 1104 (s), 1079 (s), 1040 (s), 1007 (s), 997 (s), 884 (m), 804 (s), 734 (m) cm ${ }^{-1} .{ }^{1} \mathbf{H}$ NMR $\delta 4.52$ (q, $\left.J=7.0,1 \mathrm{H}, \mathrm{CHOH}\right)$, 4.28 (s, 5H, Cp), 4.13 (br t, 1H, CHCp), 4.02 (d, $J=2.0,1 \mathrm{H}, \mathrm{CHCp}), 3.97$ (d, $J=2.0,1 \mathrm{H}$, CHCp), 3.77 (t, $\left.J=6.8,2 \mathrm{H}, \mathrm{CH}_{2} \mathrm{OH}\right), 2.73$ (dt, $\left.J=12.1,6.8,1 \mathrm{H}, \mathrm{CHHCHOH}\right), 2.44(\mathrm{~m}, 1 \mathrm{H}$, $\mathrm{CHCH}_{2} \mathrm{CH}_{2} \mathrm{OH}$ ), $2.04\left(\mathrm{hex}, J=6.8,1 \mathrm{H}, \mathrm{CHHCH} \mathrm{H}_{2} \mathrm{OH}\right), 1.86(\mathrm{~m}, 3 \mathrm{H}, \mathrm{CH} H \mathrm{CHOH}+$ $\left.\mathrm{CH} \mathrm{CCH}_{2} \mathrm{OH}+\mathrm{OH}\right), 1.59$ (br s, $\left.1 \mathrm{H}, \mathrm{OH}\right) .{ }^{13} \mathbf{C}$ NMR $\delta 100.3$ (CCp), 98.3 (CCp), 70.0 (CHCp), $69.3(\mathrm{CHOH}), 68.3(\mathrm{Cp}), 61.8\left(\mathrm{CH}_{2} \mathrm{OH}\right), 60.7(\mathrm{CHCp}), 58.9(\mathrm{CHCp}), 47.1\left(\mathrm{CH}_{2} \mathrm{CHOH}\right), 38.7$ $\left(\mathrm{CH}_{2} \mathrm{CH}_{2} \mathrm{OH}\right), 32.2\left(\mathrm{CHCH}_{2} \mathrm{CH}_{2} \mathrm{OH}\right) . \mathbf{M S}(\mathrm{EI}, 70 \mathrm{eV}) \mathrm{m} / z 313$ ([M+1] $\left.]^{+}, 17\right), 312\left([\mathrm{M}]^{+}, 91\right)$, 284 (10), $247\left([\mathrm{M}-(\mathrm{Cp})]^{+}, 23\right), 239\left(\left[\mathrm{M}-\left(\mathrm{CH}_{2} \mathrm{CO}_{2} \mathrm{Me}\right)\right]^{+}, 18\right), 215$ (24), 188 ([M-(Cp)$\left.\left.\left(\mathrm{CO}_{2} \mathrm{Me}\right)\right]^{+}, 7\right), 175(19), 121\left([\mathrm{FeCp}]^{+}, 100\right), 103(31), 56\left([\mathrm{Fe}]^{+}, 98\right)$.

\section{II.12. Synthesis of 16 via selective mono-protection of 15.}

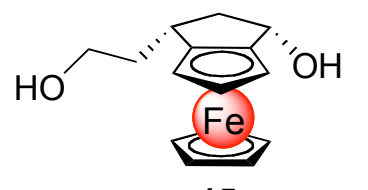

15

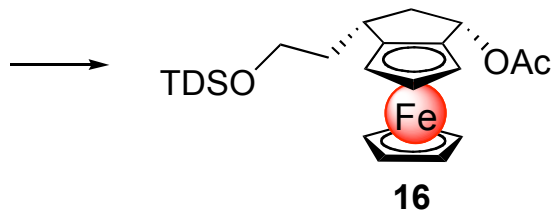

16

To a solution of diol 15 (80 mg, 1 equiv., $0.28 \mathrm{mmol}$ ) and DMAP (3.6 mg, 0.1 equiv., 0.03 $\mathrm{mmol})$ in pyridine $(2.8 \mathrm{~mL})$ was added dropwise $\mathrm{TDSCl}(56 \mu \mathrm{L}, 1$ equiv., $0.28 \mathrm{mmol})$ under argon atmosphere. After $2 \mathrm{~h}$ at room temperature, water and then $1 \mathrm{~N} \mathrm{HCl}$ were added successively. The product was extracted with MTBE and the organic phase was washed with 
brine, dried over $\mathrm{MgSO}_{4}$, filtered and concentrated under vacuum. The crude alcohol was dissolved in dry pyridine $(1.7 \mathrm{~mL})$ under argon atmosphere and acetic anhydride $(640 \mu \mathrm{L})$ was added dropwise. After stirring overnight at room temperature, a saturated aqueous solution of $\mathrm{NH}_{4} \mathrm{Cl}$ was added. The acetate was extracted with MTBE and the organic layer was washed successively with saturated aqueous $\mathrm{NaHCO}_{3}$ and brine. The organic solution was then dried with $\mathrm{Na}_{2} \mathrm{SO}_{4}$, filtered and concentrated under vacuum. Purification by flash chromatography (cyclohexane/ethyl acetate 4/1) afforded 16 as a yellow oil (113 mg, 0.24 mmol, $86 \%$ for 2 steps). $[\alpha]^{\mathbf{2 0}}{ }_{\mathbf{D}}-87.4,[\alpha]^{20}{ }_{546}-115.2,[\alpha]_{365}^{20}-156.4\left(c 0.385, \mathrm{CHCl}_{3}\right)$. IR (neat) $v 2955(\mathrm{~m}), 2860(\mathrm{w}), 1734(\mathrm{C}=$ Ost, s), $1463(\mathrm{w}), 1367(\mathrm{w}), 1237(\mathrm{~s}), 1094(\mathrm{~s}), 1036$ (m), $816(\mathrm{~s}), 776(\mathrm{~m}), 613(\mathrm{w}) \mathrm{cm}^{-1} .{ }^{1} \mathbf{H}$ NMR $\delta 5.44(\mathrm{t}, J=7.3,1 \mathrm{H}, \mathrm{CHOAc}), 4.22(\mathrm{~s}, 5 \mathrm{H}$, Cp), 4.09 (t, $J=2.2,1 \mathrm{H}, \mathrm{CHCp}), 3.95$ (d, $J=2.2,1 \mathrm{H}, \mathrm{CHCp}), 3.74$ (d, $J=2.1,1 \mathrm{H}, \mathrm{CHCp})$, $3.72\left(\mathrm{t}, J=6.6,2 \mathrm{H}, \mathrm{CH}_{2} \mathrm{O}\right), 2.74(\mathrm{dt}, J=12.2,7.0,1 \mathrm{H}, \mathrm{CHHCHOAc}), 2.53(\mathrm{~m}, 1 \mathrm{H}$, $\left.\mathrm{CHCH}_{2} \mathrm{CH}_{2} \mathrm{O}\right), 2.15\left(\mathrm{~s}, 3 \mathrm{H}, \mathrm{CH}_{3} \mathrm{CO}\right), 2.03\left(\mathrm{~m}, 2 \mathrm{H}, \mathrm{CH} H \mathrm{CHOAc}+\mathrm{CH} H \mathrm{CH}_{2} \mathrm{O}\right), 1.85(\mathrm{~m}, 1 \mathrm{H}$, $\left.\mathrm{CHHCH}_{2} \mathrm{O}\right), 1.63$ (hep, $\left.J=6.8,1 \mathrm{H}, \mathrm{CH}\left(\mathrm{CH}_{3}\right)_{2}\right), 0.88\left(\mathrm{~d}, J=6.9,6 \mathrm{H},\left(\mathrm{CH}_{3}\right)_{2} \mathrm{CH}\right), 0.85(\mathrm{~s}, 6 \mathrm{H}$, $\left.\left(\mathrm{CH}_{3}\right)_{2} \mathrm{C}\right), 0.11\left(\mathrm{~s}, 6 \mathrm{H}, \mathrm{CH}_{3} \mathrm{Si}\right) .{ }^{13} \mathrm{C}$ NMR $\delta 170.9(\mathrm{C}=\mathrm{O}), 101.1$ (CCp), 93.0 (CCp), 72.6 (CHOAc), 69.9 (CHCp), $68.5(\mathrm{Cp}), 61.7\left(\mathrm{CH}_{2} \mathrm{O}\right), 60.4$ (CHCp), 60.2 (CHCp), 42.3

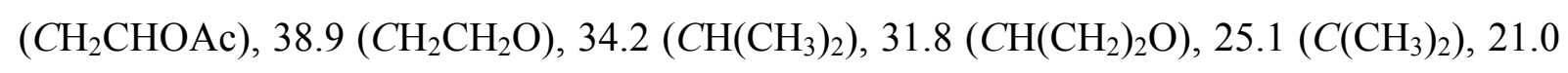
$\left(\mathrm{CH}_{3} \mathrm{CO}\right), 20.4\left(\left(\mathrm{CH}_{3}\right)_{2} \mathrm{CH}\right), 18.5\left(\left(\mathrm{CH}_{3}\right)_{2} \mathrm{C}\right),-3.34\left(\mathrm{CH}_{3} \mathrm{Si}\right),-3.37\left(\mathrm{CH}_{3} \mathrm{Si}\right)$. MS (EI, $\left.70 \mathrm{eV}\right)$ $m / z 470\left([\mathrm{M}]^{+}, 26\right), 410$ (100), 250 (39), 133 (20). HRMS (EI, $\left.70 \mathrm{eV}\right)$ calcd. for $\mathrm{C}_{25} \mathrm{H}_{38} \mathrm{FeO}_{3} \mathrm{Si} 470.1940$. Found 470.194 .

\section{II.13. Synthesis of the cytosine derivative 17.}

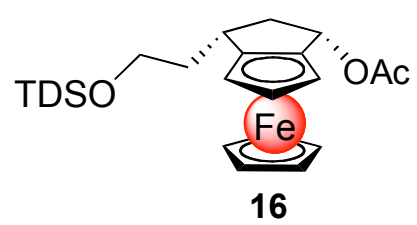

16

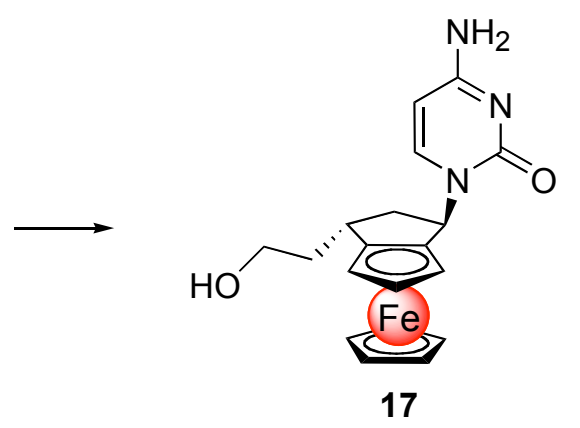

17

The general procedure for nucleobase introduction was used (see II.6.). Amounts: acetate 16 (85 mg, 1 equiv., $0.18 \mathrm{mmol}$ ), (TMS) $)_{2}$ cytosine (186 mg, 4 equiv., $0.72 \mathrm{mmol}$ ), in $\mathrm{CH}_{2} \mathrm{Cl}_{2}$ (4 $\mathrm{mL})$, TMSOTf (195 $\mu \mathrm{L}, 6$ equiv., $1.08 \mathrm{mmol})$, stirred for $0.5 \mathrm{~h}$. Flash chromatography (ethyl acetate/methanol 4/1) afforded the deprotected alcohol 17 as a major product, yellow solid (48 $\mathrm{mg}, 0.13 \mathrm{mmol}, 75 \%$ ) and a little amount of the TDS-protected ferrocenyl nucleoside analog 
18, yellow solid (3 mg, $0.006 \mathrm{mmol}, 3$ \%). Mp: decomposition at $240^{\circ} \mathrm{C} .[\mathbf{\alpha}]^{\mathbf{2 0}} \mathbf{D}-196,[\alpha]^{20}{ }_{546}$ -275 (c 0.255, EtOH). IR (neat) $v 3328$ (OHst, m), 3180 (NHst, m), 2924 (w), 2860 (w), 1635 (C=Ost, s), 1602 (s), 1521 (m), 1482 (s), 1395 (m), 1276 (s), 1184 (w), 1105 (w), 1051 (w), $999(\mathrm{w}), 787(\mathrm{~m}) \mathrm{cm}^{-1} .{ }^{1} \mathbf{H}$ NMR $\left(\mathrm{CD}_{3} \mathrm{OD}\right) \delta 6.86(\mathrm{~d}, J=7.4,1 \mathrm{H}, \mathrm{NCH}=\mathrm{CHC}), 5.70(\mathrm{~d}, J=$ 7.4, 1H, $\mathrm{NCH}=\mathrm{CHC}), 5.66(\mathrm{~d}, J=6.4,1 \mathrm{H}, \mathrm{CHN}), 4.29(\mathrm{t}, J=2.2,1 \mathrm{H}, \mathrm{CHCp}), 4.25(\mathrm{~s}, 5 \mathrm{H}$, Cp), 4.20 (d, $J=2.2,1 \mathrm{H}, \mathrm{CHCp}), 4.18$ (d, $J=2.2,1 \mathrm{H}, \mathrm{CHCp}), 3.73$ (br t, 2H, $\mathrm{CH}_{2} \mathrm{OH}$ ), 2.72 (m, 2H, CHHCHN + $\left.\mathrm{CHCH}_{2} \mathrm{CH}_{2} \mathrm{OH}\right), 2.35(\mathrm{~m}, 1 \mathrm{H}, \mathrm{CH} H \mathrm{CHN}), 2.13$ (hex, $J=6.6,1 \mathrm{H}$, $\left.\mathrm{CHHCH}_{2} \mathrm{OH}\right), 1.91$ (hex, $\left.J=6.8,1 \mathrm{H}, \mathrm{CHHCH} \mathrm{H}_{2} \mathrm{OH}\right) .{ }^{13} \mathbf{C} \mathbf{N M R}\left(\mathrm{CD}_{3} \mathrm{OD}\right) \delta 167.2\left(\mathrm{NH}_{2}-\right.$ $\mathrm{C}=\mathrm{N}), 159.0(\mathrm{C}=\mathrm{O}), 143.3(\mathrm{NCH}=\mathrm{CHC}), 103.1(\mathrm{CCp}), 95.7(\mathrm{NCH}=C \mathrm{HC}), 90.0(\mathrm{CCp}), 76.6$ (CHCp), 70.4 (Cp), 63.1 (CHCp), 62.8 (CHCp), $61.8\left(\mathrm{CH}_{2} \mathrm{OH}\right), 59.0(\mathrm{CHN}), 46.5$ $\left(\mathrm{CH}_{2} \mathrm{CHN}\right), 39.4\left(\mathrm{CH}_{2} \mathrm{CH}_{2} \mathrm{OH}\right), 33.5\left(\mathrm{CHCH}_{2} \mathrm{CH}_{2} \mathrm{OH}\right)$. MS (EI, $\left.70 \mathrm{eV}\right) \mathrm{m} / z 379\left([\mathrm{M}]^{+}, 8\right)$, 314 ([M-(Cp) $\left.]^{+}, 2\right), 269$ ([M-(cytosine) $]^{+}$and/or [M-(Cp)- $\left.\left.\left(\mathrm{CH}_{2} \mathrm{CH}_{2} \mathrm{OH}\right)\right]^{+}, 21\right), 268$ ([M(cytosine)-1 $]^{+}$and/or [M-(Cp)- $\left.\left(\mathrm{CH}_{2} \mathrm{CH}_{2} \mathrm{OH}\right)-1\right]^{+}$, 100), 231 (27), 210 (17), 172 (19), 137 (18), $121\left([\mathrm{FeCp}]^{+}, 43\right), 56\left([\mathrm{Fe}]^{+}, 29\right)$. HRMS (EI, $70 \mathrm{eV}$ ) calcd. for $\mathrm{C}_{19} \mathrm{H}_{21} \mathrm{FeN}_{3} \mathrm{O}_{2}$ 379.0983. Found 379.098 .

\section{II.14. Synthesis of the cytosine derivative 18.}

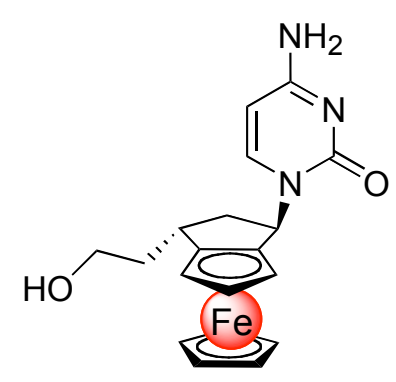

17

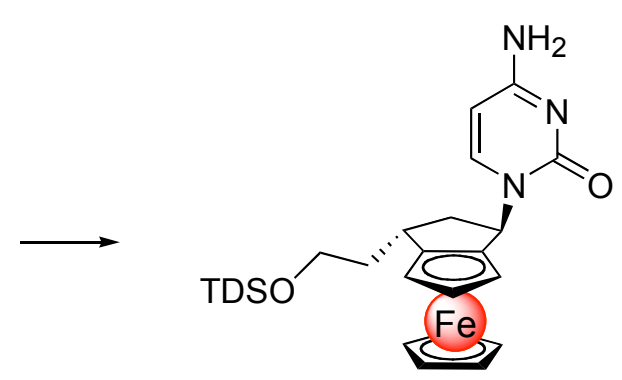

18

Under argon atmosphere, the alcohol 17 (32 mg, 1 equiv., $0.084 \mathrm{mmol})$, imidazole (14 $\mathrm{mg}$, 2.4 equiv., $0.20 \mathrm{mmol})$ and DMAP $(0.9 \mathrm{mg}, 0.09$ equiv., $0.008 \mathrm{mmol})$ were dissolved in dry DMF $(800 \mu \mathrm{L})$. TDSCl $(20 \mu \mathrm{L}, 1.2$ equiv., $0.100 \mathrm{mmol})$ was added dropwise and the mixture was stirred overnight at room temperature. Saturated aqueous $\mathrm{NH}_{4} \mathrm{Cl}$ was then added and the product was extracted with $\mathrm{CH}_{2} \mathrm{Cl}_{2}$. The organic layer was washed with brine, dried over $\mathrm{Na}_{2} \mathrm{SO}_{4}$, filtered and concentrated under vacuum. Purification by flash chromatography (ethyl acetate then ethyl acetate/methanol 95/5) afforded 18 as a yellow powder (40 $\mathrm{mg}, 0.077$ mmol, 92\%). Mp: decomposition starts when $\mathrm{T}>200^{\circ} \mathrm{C}$. $[\alpha]^{\mathbf{2 0}}{ }_{\mathbf{D}}-174,[\alpha]_{546}^{20}-241(c 0.30$, $\mathrm{CHCl}_{3}$ ). IR (neat) $v 3349$ (w), 3146 (w), 3092 (w), 2953 (m), 2860 (m), 1643 (s), 1624 (s), 1517 (m), 1484 (s), 1392 (m), 1277 (m), 1249 (m), 1105 (m), 829 (m), 776 (m) cm ${ }^{-1} .{ }^{1} \mathbf{H}$ 
NMR $\delta 6.68(\mathrm{~d}, J=7.7,1 \mathrm{H}, \mathrm{NCH}=\mathrm{CHC}), 6.60-5.80\left(\right.$ br s, $\left.2 \mathrm{H}, \mathrm{NH}_{2}\right), 5.69(\mathrm{~d}, J=6.5,1 \mathrm{H}$, $\mathrm{CHN}), 5.52(\mathrm{~d}, J=7.3,1 \mathrm{H}, \mathrm{NCH}=\mathrm{CHC}), 4.20(\mathrm{t}, J=2.3,1 \mathrm{H}, \mathrm{CHCp}), 4.17(\mathrm{~s}, 5 \mathrm{H}, \mathrm{Cp}), 4.08$ (d, $J=2.2,1 \mathrm{H}, \mathrm{CHCp}), 4.05$ (d, $J=2.2,1 \mathrm{H}, \mathrm{CHCp}), 3.68\left(\mathrm{t}, J=6.7,2 \mathrm{H}, \mathrm{CH}_{2} \mathrm{O}\right), 2.61$ (m, $\left.2 \mathrm{H}, \mathrm{CHHCHN}+\mathrm{CHCH}_{2} \mathrm{CHN}\right), 2.34(\mathrm{dd}, J=12.3,5.7,1 \mathrm{H}, \mathrm{CH} H \mathrm{CHN}), 2.00(\mathrm{hex}, J=6.6$, $1 \mathrm{H}, \mathrm{C} H \mathrm{HCH}_{2} \mathrm{O}$ ), $1.82\left(\right.$ hex, $\left.J=6.6,1 \mathrm{H}, \mathrm{CH} H \mathrm{CH}_{2} \mathrm{O}\right), 1.58\left(\mathrm{sep}, J=7.0,1 \mathrm{H}, \mathrm{CH}\left(\mathrm{CH}_{3}\right)_{2}\right), 0.83$ $\left(\mathrm{d}, J=6.8,6 \mathrm{H},\left(\mathrm{CH}_{3}\right)_{2} \mathrm{CH}\right), 0.81\left(\mathrm{~s}, 6 \mathrm{H},\left(\mathrm{CH}_{3}\right)_{2} \mathrm{C}\right), 0.08\left(\mathrm{~s}, 3 \mathrm{H}, \mathrm{CH}_{3} \mathrm{Si}\right), 0.07\left(\mathrm{~s}, 3 \mathrm{H}, \mathrm{CH}_{3} \mathrm{Si}\right)$. ${ }^{13}$ C NMR $\delta 165.3\left(\mathrm{NH}_{2}-\mathrm{C}=\mathrm{N}\right), 156.6(\mathrm{C}=\mathrm{O}), 142.0(\mathrm{NCH}=\mathrm{CHC}), 101.8(\mathrm{CCp}), 93.4$ $(\mathrm{NCH}=\mathrm{CHC}), 88.8(\mathrm{CCp}), 71.1(\mathrm{CHCp}), 69.2(\mathrm{Cp}), 61.7(2 \times \mathrm{CHCp}), 61.6\left(\mathrm{CH}_{2} \mathrm{O}\right), 57.2$ $(\mathrm{CHN}), 45.4\left(\mathrm{CH}_{2} \mathrm{CHN}\right), 38.2\left(\mathrm{CH}_{2} \mathrm{CH}_{2} \mathrm{O}\right), 34.2\left(\mathrm{CH}_{\left.\left(\mathrm{CH}_{3}\right)_{2}\right),} 32.0\left(\mathrm{CH}\left(\mathrm{CH}_{2}\right)_{2} \mathrm{O}\right), 25.1\right.$ $\left(C\left(\mathrm{CH}_{3}\right)_{2}\right), 20.4\left(\left(\mathrm{CH}_{3}\right)_{2} \mathrm{CH}\right), 18.5\left(\left(\mathrm{CH}_{3}\right)_{2} \mathrm{C}\right),-3.4\left(\mathrm{CH}_{3} \mathrm{Si}\right)$. MS (EI, $\left.70 \mathrm{eV}\right) \mathrm{m} / z 521\left([\mathrm{M}]^{+}\right.$, 5), 411 ([M-(cytosine) $]^{+}$and/or $\left.\left[\mathrm{M}-(\mathrm{Cp})-\left(\mathrm{CH}_{2} \mathrm{CH}_{2} \mathrm{OH}\right)\right]^{+}, 32\right), 410$ ([M-(cytosine)-1 $]^{+}$. and/or [M-(Cp)-( $\left.\left.\left.\mathrm{CH}_{2} \mathrm{CH}_{2} \mathrm{OH}\right)-1\right]^{+}, 100\right), 250$ (100), 231 (97), 168 (37), 121 ([FeCp] , 79), 73 (82).

HRMS (EI, $70 \mathrm{eV}$ ) calcd. for $\mathrm{C}_{27} \mathrm{H}_{39} \mathrm{FeN}_{3} \mathrm{O}_{2} \mathrm{Si}$ 521.2161. Found 521.216. 


\section{X-ray Crystallographic Data for Compound 6}
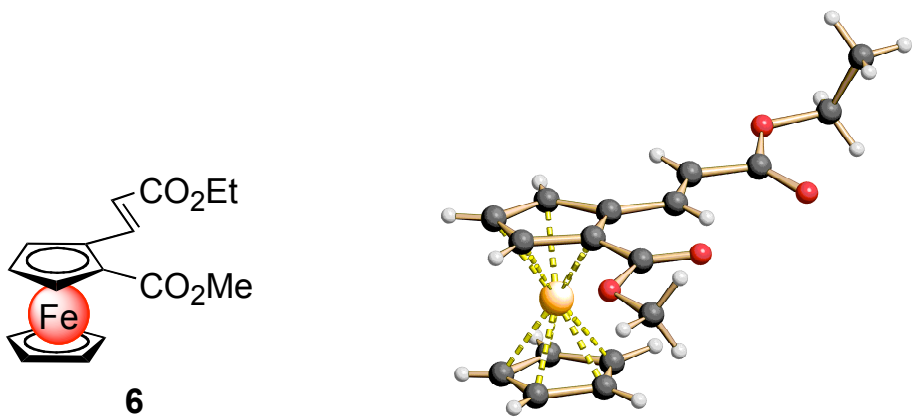

Table 1. Crystal data and structure refinement for $\mathbf{6}$.

Identification code

Empirical formula

Formula weight

Temperature

Wavelength

Crystal system, space group

Unit cell dimensions

Volume

Z, Calculated density

Absorption coefficient

$\mathrm{F}(000)$

Crystal size

Theta range for data collection

Limiting indices

Reflections collected / unique

Reflection observed [I $>2$ sigma(I)

Completeness to theta $=26.99$

Absorption correction

Refinement method

Data / restraints / parameters

Goodness-of-fit on $\mathrm{F}^{\wedge} 2$

Final $\mathrm{R}$ indices [I $>2 \operatorname{sigma}(\mathrm{I})]$

$\mathrm{R}$ indices (all data)

Absolute structure parameter

Largest diff. peak and hole
Z_pj193

C17 H18 Fe O4

342.16

100(2) K

$0.71073 \mathrm{~A}$

Monoclinic, P21

$\mathrm{a}=9.7914(6) \mathrm{A}$ alpha $=90 \mathrm{deg}$.

$\mathrm{b}=8.0967(3)$ A $\quad$ beta $=114.960(2) \mathrm{deg}$.

$\mathrm{c}=10.6853(7) \mathrm{A}$ gamma $=90 \mathrm{deg}$.

767.99(7) $\mathrm{A}^{\wedge} 3$

2, $1.480 \mathrm{Mg} / \mathrm{m}^{\wedge} 3$

$0.997 \mathrm{~mm}^{\wedge}-1$

356

$3 \times .2 \times .2 \mathrm{~mm}$

2.10 to $26.99 \mathrm{deg}$.

$-10<=\mathrm{h}<=12,-8<=\mathrm{k}<=10,-11<=\mathrm{l}<=13$

$3908 / 3053[\mathrm{R}($ int $)=0.0270]$

I) 2640

$99.6 \%$

None

Full-matrix least-squares on $\mathrm{F}^{\wedge} 2$

3053 / 1 / 249

0.967

$\mathrm{R} 1=0.0365, \mathrm{wR} 2=0.0669$

$\mathrm{R} 1=0.0465, \mathrm{wR} 2=0.0694$

$0.004(17)$

0.268 and -0.310 e. $\mathrm{A}^{\wedge}-3$ 
Table 2. Atomic coordinates ( $\left.\times 10^{\wedge} 4\right)$ and equivalent isotropic displacement parameters $\left(\mathrm{A}^{\wedge} 2 \times 10^{\wedge} 3\right)$ for 6 .

$\mathrm{U}(\mathrm{eq})$ is defined as one third of the trace of the orthogonalized Uij tensor.

\begin{tabular}{lcccc}
\hline & $\mathrm{x}$ & $\mathrm{z}$ & $\mathrm{U}(\mathrm{eq})$ \\
& & & \\
$\mathrm{Fe}(1)$ & $7111(1)$ & $904(1)$ & $2796(1)$ & $18(1)$ \\
$\mathrm{C}(1)$ & $1232(3)$ & $736(4)$ & $33(3)$ & $17(1)$ \\
$\mathrm{O}(3)$ & $793(2)$ & $409(2)$ & $906(2)$ & $22(1)$ \\
$\mathrm{O}(4)$ & $331(2)$ & $1316(2)$ & $-1236(2)$ & $20(1)$ \\
$\mathrm{O}(5)$ & $7519(3)$ & $-2996(3)$ & $4651(2)$ & $26(1)$ \\
$\mathrm{C}(6)$ & $5377(4)$ & $-520(3)$ & $1511(3)$ & $16(1)$ \\
$\mathrm{C}(7)$ & $3820(4)$ & $-233(3)$ & $1266(3)$ & $16(1)$ \\
$\mathrm{C}(8)$ & $6472(4)$ & $-1484(4)$ & $2603(4)$ & $17(1)$ \\
$\mathrm{C}(9)$ & $6225(4)$ & $-2403(4)$ & $3692(3)$ & $20(1)$ \\
$\mathrm{C}(10)$ & $2788(3)$ & $554(4)$ & $190(3)$ & $19(1)$ \\
$\mathrm{C}(11)$ & $7889(4)$ & $-1347(4)$ & $2510(4)$ & $21(1)$ \\
$\mathrm{O}(12)$ & $5024(3)$ & $-2606(3)$ & $3727(2)$ & $32(1)$ \\
$\mathrm{C}(13)$ & $7674(4)$ & $-307(4)$ & $1382(4)$ & $23(1)$ \\
$\mathrm{C}(14)$ & $6152(4)$ & $195(4)$ & $770(3)$ & $20(1)$ \\
$\mathrm{C}(15)$ & $7416(4)$ & $-3924(5)$ & $5762(3)$ & $31(1)$ \\
$\mathrm{C}(16)$ & $-1263(4)$ & $1475(4)$ & $-1547(4)$ & $19(1)$ \\
$\mathrm{C}(1 \mathrm{~A})$ & $-2054(4)$ & $-159(4)$ & $-1997(4)$ & $29(1)$ \\
$\mathrm{C}(2 \mathrm{~A})$ & $6207(5)$ & $2757(4)$ & $3499(4)$ & $31(1)$ \\
$\mathrm{C}(3 \mathrm{~A})$ & $8594(5)$ & $1803(5)$ & $4660(5)$ & $45(1)$ \\
$\mathrm{C}(4 \mathrm{~A})$ & $7128(5)$ & $1785(4)$ & $4585(4)$ & $34(1)$ \\
$\mathrm{C}(5 \mathrm{~A})$ & $8551(6)$ & $2825(6)$ & $3577(6)$ & $55(2)$ \\
$\mathrm{C}(6 \mathrm{~A})$ & $7079(6)$ & $3422(5)$ & $2869(5)$ & $42(1)$ \\
& & & & \\
\hline
\end{tabular}


Table 3. Anisotropic displacement parameters $\left(\mathrm{A}^{\wedge} 2 \times 10^{\wedge} 3\right)$ for 6.

The anisotropic displacement factor exponent takes the form:

$-2 \mathrm{pi}^{\wedge} 2\left[\mathrm{~h}^{\wedge} 2 \mathrm{a}^{* \wedge} 2 \mathrm{U} 11+\ldots+2 \mathrm{~h} \mathrm{k} \mathrm{a}{ }^{*} \mathrm{~b}^{*} \mathrm{U} 12\right]$

\begin{tabular}{|c|c|c|c|c|c|c|}
\hline & U11 & U22 & U33 & U23 & U13 & U12 \\
\hline $\mathrm{Fe}(1)$ & $16(1)$ & $19(1)$ & $19(1)$ & $-2(1)$ & $9(1)$ & $-1(1)$ \\
\hline $\mathrm{C}(1)$ & $16(1)$ & $13(2)$ & $21(2)$ & $-3(2)$ & $7(1)$ & $-2(2)$ \\
\hline $\mathrm{O}(3)$ & $18(1)$ & $30(2)$ & $21(1)$ & $3(1)$ & $10(1)$ & $2(1)$ \\
\hline $\mathrm{O}(4)$ & $16(1)$ & $25(1)$ & $20(1)$ & $6(1)$ & $7(1)$ & $2(1)$ \\
\hline $\mathrm{O}(5)$ & $28(1)$ & $25(1)$ & $22(1)$ & $8(1)$ & $8(1)$ & $4(1)$ \\
\hline$C(6)$ & $17(2)$ & $19(2)$ & $13(2)$ & $-4(1)$ & $7(1)$ & $-4(1)$ \\
\hline$C(7)$ & $17(2)$ & $19(2)$ & $14(2)$ & $-3(1)$ & $8(2)$ & $-4(1)$ \\
\hline $\mathrm{C}(8)$ & $13(2)$ & $19(2)$ & $18(2)$ & $-1(1)$ & $4(2)$ & $2(1)$ \\
\hline $\mathrm{C}(9)$ & $23(2)$ & $15(2)$ & $21(2)$ & $-2(1)$ & $9(2)$ & $0(1)$ \\
\hline$C(10)$ & $19(2)$ & $22(2)$ & $18(2)$ & $1(1)$ & $9(1)$ & $-1(1)$ \\
\hline $\mathrm{C}(11)$ & $18(2)$ & $23(2)$ & $23(2)$ & $-1(1)$ & $10(2)$ & $4(2)$ \\
\hline $\mathrm{O}(12)$ & $23(1)$ & $40(1)$ & $33(2)$ & $12(1)$ & $12(1)$ & $-2(1)$ \\
\hline$C(13)$ & $20(2)$ & $30(2)$ & $24(2)$ & $-4(2)$ & $16(2)$ & $0(1)$ \\
\hline$C(14)$ & $21(2)$ & $25(2)$ & $17(2)$ & $-1(2)$ & $11(2)$ & $-1(1)$ \\
\hline$C(15)$ & $43(2)$ & $26(2)$ & $20(2)$ & $3(2)$ & $10(2)$ & $2(2)$ \\
\hline$C(16)$ & $13(2)$ & $21(2)$ & $22(2)$ & $4(1)$ & $6(2)$ & $3(1)$ \\
\hline$C(1 \mathrm{~A})$ & $24(2)$ & $25(2)$ & $28(2)$ & $1(2)$ & $1(2)$ & $-3(2)$ \\
\hline $\mathrm{C}(2 \mathrm{~A})$ & $28(2)$ & $27(2)$ & $38(2)$ & $-13(2)$ & $12(2)$ & $3(2)$ \\
\hline$C(3 A)$ & $34(3)$ & $35(2)$ & $41(3)$ & $-19(2)$ & $-10(2)$ & $5(2)$ \\
\hline$C(4 A)$ & $54(3)$ & $24(2)$ & $26(2)$ & $-8(2)$ & $20(2)$ & $-6(2)$ \\
\hline$C(5 A)$ & $47(3)$ & $49(3)$ & $89(4)$ & $-42(3)$ & $49(3)$ & $-33(2)$ \\
\hline$C(6 \mathrm{~A})$ & $77(4)$ & $15(2)$ & $31(3)$ & $-1(2)$ & $20(3)$ & $-6(2)$ \\
\hline
\end{tabular}


Table 4. Hydrogen coordinates ( x 10^4) and isotropic displacement parameters $\left(\mathrm{A}^{\wedge} 2 \times 10^{\wedge} 3\right)$ for $\mathbf{6}$.

\begin{tabular}{|c|c|c|c|c|}
\hline & $\mathrm{x}$ & z & $\mathrm{U}(\mathrm{eq})$ & \\
\hline $\mathrm{H}(15 \mathrm{~A})$ & 7112 & -3187 & 6327 & 46 \\
\hline $\mathrm{H}(15 \mathrm{~B})$ & 8399 & -4412 & 6336 & 46 \\
\hline $\mathrm{H}(15 \mathrm{C})$ & 6667 & -4804 & 5377 & 46 \\
\hline $\mathrm{H}(1 \mathrm{~A} 1)$ & -1874 & -594 & -2770 & 44 \\
\hline $\mathrm{H}(1 \mathrm{~A} 2)$ & -3138 & -7 & -2289 & \\
\hline $\mathrm{H}(1 \mathrm{~A} 3)$ & -1664 & -940 & -1225 & 44 \\
\hline $\mathrm{H}(1)$ & $2990(30)$ & $980(50)$ & $-520(30)$ & $24(7)$ \\
\hline $\mathrm{H}(2)$ & $5750(30)$ & $880(50)$ & $-20(30)$ & $21(7)$ \\
\hline $\mathrm{H}(3)$ & $8850(40)$ & $-1850(40)$ & $3120(30)$ & $24(9)$ \\
\hline $\mathrm{H}(4)$ & $-1400(30)$ & $1810(30)$ & $-760(30)$ & $15(8)$ \\
\hline $\mathrm{H}(5)$ & $8440(40)$ & $-100(40)$ & $1140(30)$ & $33(10)$ \\
\hline $\mathrm{H}(6)$ & $3590(40)$ & $-670(40)$ & $1900(30)$ & $19(8)$ \\
\hline $\mathrm{H}(7)$ & $9510(50)$ & $1070(70)$ & $5290(40)$ & $80(14)$ \\
\hline $\mathrm{H}(9)$ & $-1650(30)$ & $2360(40)$ & $-2320(30)$ & $12(8)$ \\
\hline $\mathrm{H}(3 \mathrm{~A})$ & $5050(50)$ & $2920(50)$ & $3100(40)$ & $64(13)$ \\
\hline $\mathrm{H}(7 \mathrm{~A})$ & $6810(50)$ & $4130(60)$ & $2090(40)$ & $69(14)$ \\
\hline $\mathrm{H}(5 \mathrm{~A})$ & $6860(40)$ & $1230(50)$ & $5240(40)$ & $46(11)$ \\
\hline $\mathrm{H}(9 \mathrm{~A})$ & $9240(60)$ & $3050(60)$ & $3400(50)$ & $81(19)$ \\
\hline
\end{tabular}


X-ray Crystallographic Data for Compound 15

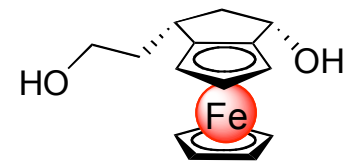

15

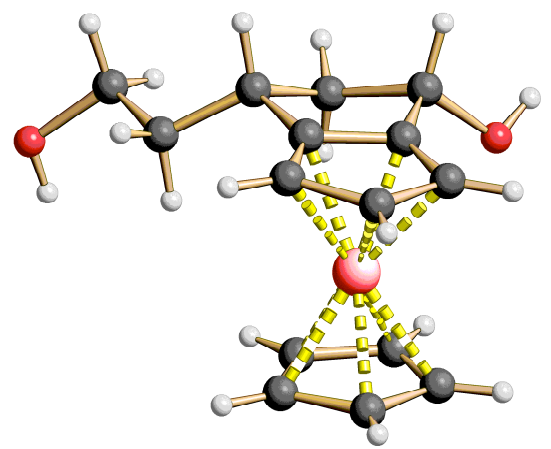

Table 5. Crystal data and structure refinement for $\mathbf{1 5 .}$

Identification code

Empirical formula

Formula weight

Temperature

Wavelength

Crystal system, space group

Unit cell dimensions

Volume

Z, Calculated density

Absorption coefficient

$\mathrm{F}(000)$

Crystal size

Theta range for data collection

Limiting indices

Reflections collected / unique

Reflection observed [I $>2$ sigma(I)

Completeness to theta $=26.99$

Absorption correction

Refinement method

Data / restraints / parameters

Goodness-of-fit on $\mathrm{F}^{\wedge} 2$

Final $\mathrm{R}$ indices [I $>2 \operatorname{sigma}(\mathrm{I})]$

$\mathrm{R}$ indices (all data)

Absolute structure parameter

Largest diff. peak and hole pj213

$\mathrm{C} 15 \mathrm{H} 18 \mathrm{Fe} \mathrm{O} 2$

286.14

$100(2) \mathrm{K}$

$0.71073 \mathrm{~A}$

Orthorhombic, P212121

$\mathrm{a}=7.8262(5)$ A alpha $=90 \mathrm{deg}$.

$\mathrm{b}=12.4546(10)$ A beta $=90 \mathrm{deg}$.

$\mathrm{c}=26.589(2) \mathrm{A}$ gamma $=90 \mathrm{deg}$.

2591.7(3) $\mathrm{A}^{\wedge} 3$

$8,1.467 \mathrm{Mg} / \mathrm{m}^{\wedge} 3$

$1.154 \mathrm{~mm}^{\wedge}-1$

1200

$.3 \times .1 \times .1 \mathrm{~mm}$

2.24 to $26.99 \mathrm{deg}$.

$-9<=\mathrm{h}<=6,-15<=\mathrm{k}<=14,-15<=1<=33$

$8433 / 5113$ [R(int) $=0.0522]$

I)] 2996

$96.5 \%$

None

Full-matrix least-squares on $\mathrm{F}^{\wedge} 2$

5113 / 0 / 329

0.894

$\mathrm{R} 1=0.0460, \mathrm{wR} 2=0.0759$

$\mathrm{R} 1=0.1063, \mathrm{wR} 2=0.0881$

$0.007(19)$

0.536 and -0.460 e. $\mathrm{A}^{\wedge}-3$ 
Table 6. Atomic coordinates ( $\times 10^{\wedge} 4$ ) and equivalent isotropic displacement parameters $\left(\mathrm{A}^{\wedge} 2 \times 10^{\wedge} 3\right)$ for 15.

$\mathrm{U}(\mathrm{eq})$ is defined as one third of the trace of the orthogonalized Uij tensor.

\begin{tabular}{lcccc}
\hline & $\mathrm{x}$ & $\mathrm{z}$ & $\mathrm{U}(\mathrm{eq})$ & \\
& & & & \\
$\mathrm{Fe}(2)$ & $9034(1)$ & $7602(1)$ & $2028(1)$ & $37(1)$ \\
$\mathrm{O}(1)$ & $7350(4)$ & $8359(3)$ & $3254(1)$ & $38(1)$ \\
$\mathrm{C}(2)$ & $6782(5)$ & $7627(4)$ & $2411(2)$ & $33(1)$ \\
$\mathrm{C}(4)$ & $7321(6)$ & $6560(4)$ & $2336(2)$ & $40(1)$ \\
$\mathrm{C}(9)$ & $5881(5)$ & $9249(3)$ & $1989(2)$ & $35(1)$ \\
$\mathrm{C}(11)$ & $11262(6)$ & $7244(4)$ & $2380(2)$ & $51(2)$ \\
$\mathrm{C}(12)$ & $10856(6)$ & $8326(4)$ & $2457(2)$ & $43(1)$ \\
$\mathrm{C}(18)$ & $6540(5)$ & $8126(4)$ & $1936(2)$ & $37(1)$ \\
$\mathrm{C}(19)$ & $6251(6)$ & $8388(4)$ & $2818(2)$ & $35(1)$ \\
$\mathrm{C}(21)$ & $5969(6)$ & $11194(4)$ & $1670(2)$ & $43(1)$ \\
$\mathrm{C}(22)$ & $10749(5)$ & $8856(4)$ & $1988(2)$ & $46(1)$ \\
$\mathrm{O}(25)$ & $6896(3)$ & $11922(3)$ & $1356(1)$ & $46(1)$ \\
$\mathrm{C}(30)$ & $6982(5)$ & $7372(4)$ & $1558(2)$ & $41(1)$ \\
$\mathrm{C}(31)$ & $7458(6)$ & $6401(4)$ & $1801(2)$ & $44(1)$ \\
$\mathrm{C}(32)$ & $6295(5)$ & $9493(4)$ & $2553(2)$ & $37(1)$ \\
$\mathrm{C}(34)$ & $11447(6)$ & $7080(5)$ & $1850(2)$ & $56(2)$ \\
$\mathrm{C}(38)$ & $11120(6)$ & $8064(5)$ & $1621(2)$ & $54(2)$ \\
$\mathrm{C}(42)$ & $6622(6)$ & $10048(4)$ & $1614(2)$ & $42(1)$ \\
$\mathrm{Fe}(1)$ & $2509(1)$ & $5462(1)$ & $352(1)$ & $36(1)$ \\
$\mathrm{C}(3)$ & $222(6)$ & $5495(5)$ & $-13(2)$ & $50(2)$ \\
$\mathrm{C}(5)$ & $4944(5)$ & $5520(4)$ & $62(2)$ & $33(1)$ \\
$\mathrm{O}(6)$ & $5443(3)$ & $3317(3)$ & $-1490(1)$ & $44(1)$ \\
$\mathrm{C}(10)$ & $4186(6)$ & $6139(4)$ & $846(2)$ & $53(2)$ \\
$\mathrm{C}(13)$ & $4979(5)$ & $5285(4)$ & $585(2)$ & $37(1)$ \\
$\mathrm{C}(14)$ & $4118(6)$ & $6518(4)$ & $-3(2)$ & $50(1)$ \\
$\mathrm{C}(15)$ & $5932(5)$ & $4700(4)$ & $-238(2)$ & $34(1)$ \\
& $985(5)$ & $4474(4)$ & $-70(2)$ & $42(1)$ \\
& & & &
\end{tabular}




$\begin{array}{llccc}\mathrm{C}(17) & 624(5) & 4779(4) & 770(2) & 44(1) \\ \mathrm{O}(20) & 5031(3) & 3563(3) & 1016(1) & 55(1) \\ \mathrm{C}(23) & -10(5) & 5676(4) & 512(2) & 48(2) \\ \mathrm{C}(24) & 3622(6) & 6899(4) & 484(2) & 64(2) \\ \mathrm{C}(26) & 6344(5) & 3787(4) & -1074(2) & 41(1) \\ \mathrm{C}(29) & 5107(5) & 4351(4) & -728(2) & 39(1) \\ \mathrm{C}(35) & 1228(5) & 4037(3) & 417(2) & 41(1) \\ \mathrm{C}(36) & 5904(6) & 4264(4) & 681(2) & 42(1) \\ \mathrm{C}(1 \mathrm{~A}) & 6126(6) & 3774(4) & 152(2) & 38(1)\end{array}$

Table 7. Anisotropic displacement parameters $\left(\mathrm{A}^{\wedge} 2 \times 10^{\wedge} 3\right)$ for 15.

The anisotropic displacement factor exponent takes the form:

$-2 \mathrm{pi}^{\wedge} 2\left[\mathrm{~h}^{\wedge} 2 \mathrm{a}^{* \wedge} 2 \mathrm{U} 11+\ldots+2 \mathrm{~h} \mathrm{k} \mathrm{a} \mathrm{a}^{*} \mathrm{U} 12\right]$

\begin{tabular}{lllllll}
\hline & $\mathrm{U} 11$ & $\mathrm{U} 22$ & $\mathrm{U} 33$ & $\mathrm{U} 23$ & $\mathrm{U} 13$ & $\mathrm{U} 12$ \\
$\mathrm{Fe}(2)$ & $39(1)$ & $48(1)$ & $25(1)$ & $-3(1)$ & $2(1)$ & $2(1)$ \\
$\mathrm{O}(1)$ & $39(2)$ & $56(2)$ & $19(2)$ & $-2(2)$ & $0(2)$ & $8(2)$ \\
$\mathrm{C}(2)$ & $41(3)$ & $34(3)$ & $25(3)$ & $-1(3)$ & $2(2)$ & $-5(2)$ \\
$\mathrm{C}(4)$ & $50(3)$ & $40(3)$ & $31(3)$ & $3(3)$ & $3(3)$ & $-10(3)$ \\
$\mathrm{C}(9)$ & $36(2)$ & $43(3)$ & $27(3)$ & $4(3)$ & $2(2)$ & $1(2)$ \\
$\mathrm{C}(11)$ & $40(3)$ & $63(4)$ & $49(4)$ & $4(3)$ & $-9(2)$ & $11(3)$ \\
$\mathrm{C}(12)$ & $37(3)$ & $58(4)$ & $34(3)$ & $-6(3)$ & $6(2)$ & $-3(3)$ \\
$\mathrm{C}(18)$ & $35(3)$ & $50(3)$ & $25(3)$ & $-3(3)$ & $-1(2)$ & $-7(2)$ \\
$\mathrm{C}(19)$ & $38(3)$ & $42(3)$ & $25(3)$ & $0(2)$ & $5(2)$ & $2(2)$ \\
$\mathrm{C}(21)$ & $37(3)$ & $53(3)$ & $38(3)$ & $8(3)$ & $-2(2)$ & $-8(3)$ \\
$\mathrm{C}(22)$ & $32(3)$ & $56(3)$ & $50(3)$ & $3(4)$ & $-1(3)$ & $-11(2)$ \\
$\mathrm{O}(25)$ & $43(2)$ & $53(2)$ & $42(2)$ & $12(2)$ & $2(2)$ & $6(2)$ \\
$\mathrm{C}(30)$ & $43(3)$ & $53(3)$ & $26(3)$ & $-8(3)$ & $-2(2)$ & $-4(3)$ \\
$\mathrm{C}(31)$ & $56(3)$ & $41(3)$ & $35(3)$ & $-11(3)$ & $7(3)$ & $1(3)$ \\
$\mathrm{C}(32)$ & $41(3)$ & $39(3)$ & $31(3)$ & $-2(3)$ & $-4(2)$ & $6(2)$ \\
$\mathrm{C}(34)$ & $42(3)$ & $73(5)$ & $53(4)$ & $-9(3)$ & $10(3)$ & $7(3)$ \\
$\mathrm{C}(38)$ & $42(3)$ & $89(5)$ & $29(3)$ & $-1(3)$ & $4(3)$ & $-9(3)$ \\
& & & & & & \\
\hline
\end{tabular}




$\begin{array}{lcccccc}\mathrm{C}(42) & 42(3) & 52(3) & 31(3) & -2(3) & -4(2) & 0(2) \\ \mathrm{Fe}(1) & 37(1) & 33(1) & 39(1) & 0(1) & 5(1) & -2(1) \\ \mathrm{C}(3) & 47(3) & 49(4) & 55(4) & 11(4) & -7(3) & -3(3) \\ \mathrm{C}(5) & 37(3) & 30(3) & 33(3) & -2(3) & 4(2) & -9(3) \\ \mathrm{O}(6) & 48(2) & 60(3) & 25(2) & -9(2) & 0(2) & 14(2) \\ \mathrm{C}(10) & 41(3) & 61(4) & 55(4) & -21(3) & 13(3) & -10(3) \\ \mathrm{C}(13) & 33(3) & 45(4) & 33(3) & -12(3) & 4(2) & -4(2) \\ \mathrm{C}(14) & 53(3) & 39(3) & 60(4) & 11(3) & 23(3) & -1(3) \\ \mathrm{C}(15) & 34(3) & 38(3) & 31(3) & 2(2) & 2(2) & 6(2) \\ \mathrm{C}(16) & 37(3) & 50(3) & 39(3) & 4(3) & -4(2) & -14(3) \\ \mathrm{C}(17) & 36(3) & 47(4) & 47(3) & 5(3) & 1(2) & -4(2) \\ \mathrm{O}(20) & 42(2) & 85(3) & 37(2) & 28(2) & 11(2) & 8(2) \\ \mathrm{C}(23) & 38(3) & 37(3) & 69(4) & 6(3) & 10(3) & 9(2) \\ \mathrm{C}(24) & 60(4) & 29(3) & 102(5) & -13(4) & 28(3) & -9(3) \\ \mathrm{C}(26) & 45(3) & 54(3) & 24(3) & 4(3) & 2(2) & 3(3) \\ \mathrm{C}(29) & 36(3) & 61(4) & 20(3) & -5(3) & 1(2) & 8(2) \\ \mathrm{C}(35) & 42(3) & 26(3) & 56(4) & 6(3) & 5(3) & 3(2) \\ \mathrm{C}(36) & 34(3) & 70(4) & 22(3) & 7(3) & 4(2) & -6(3) \\ \mathrm{C}(1 \mathrm{~A}) & 41(3) & 35(3) & 37(3) & 3(2) & 1(2) & 7(2)\end{array}$

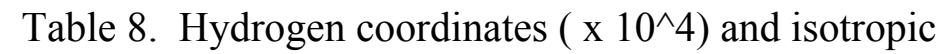
displacement parameters $\left(\mathrm{A}^{\wedge} 2 \times 10^{\wedge} 3\right)$ for $\mathbf{1 5}$.

\begin{tabular}{lcccc}
\hline \multicolumn{3}{c}{ x } & \multicolumn{2}{c}{$\mathrm{z}$} \\
& & & & \\
\hline $\mathrm{H}(1)$ & 6765 & 8461 & 3514 & 57 \\
$\mathrm{H}(4)$ & 7551 & 6043 & 2590 & 48 \\
$\mathrm{H}(9)$ & 4612 & 9236 & 1947 & 42 \\
$\mathrm{H}(11)$ & 11393 & 6714 & 2634 & 61 \\
$\mathrm{H}(12)$ & 10679 & 8653 & 2775 & 51 \\
$\mathrm{H}(19)$ & 5052 & 8223 & 2922 & 42 \\
$\mathrm{H}(21 \mathrm{~A})$ & 4742 & 11218 & 1580 & 51 \\
$\mathrm{H}(21 \mathrm{~B})$ & 6082 & 11420 & 2025 & 51
\end{tabular}




$\begin{array}{lcccc}\mathrm{H}(22) & 10483 & 9590 & 1931 & 55 \\ \mathrm{H}(25) & 7932 & 11918 & 1439 & 69 \\ \mathrm{H}(30) & 6964 & 7494 & 1206 & 49 \\ \mathrm{H}(31) & 7807 & 5758 & 1638 & 53 \\ \mathrm{H}(32 \mathrm{~A}) & 7437 & 9827 & 2586 & 45 \\ \mathrm{H}(32 \mathrm{~B}) & 5431 & 9982 & 2699 & 45 \\ \mathrm{H}(34) & 11736 & 6427 & 1687 & 68 \\ \mathrm{H}(38) & 11143 & 8187 & 1269 & 64 \\ \mathrm{H}(42 \mathrm{~A}) & 7881 & 10053 & 1650 & 50 \\ \mathrm{H}(42 \mathrm{~B}) & 6357 & 9796 & 1269 & 50 \\ \mathrm{H}(3) & -80 & 5973 & -276 & 60 \\ \mathrm{H}(6) & 6040 & 2828 & -1617 & 66 \\ \mathrm{H}(10) & 4053 & 6194 & 1200 & 63 \\ \mathrm{H}(14) & 3925 & 6873 & -314 & 61 \\ \mathrm{H}(15) & 7091 & 4997 & -313 & 41 \\ \mathrm{H}(16) & 1282 & 4140 & -379 & 51 \\ \mathrm{H}(17) & 641 & 4690 & 1125 & 52 \\ \mathrm{H}(20) & 5696 & 3072 & 1109 & 82 \\ \mathrm{H}(23) & -506 & 6296 & 661 & 58 \\ \mathrm{H}(24) & 3022 & 7545 & 553 & 76 \\ \mathrm{H}(26 \mathrm{~A}) & 7198 & 4307 & -1201 & 49 \\ \mathrm{H}(26 \mathrm{~B}) & 6958 & 3219 & -887 & 49 \\ \mathrm{H}(29 \mathrm{~A}) & 4142 & 3863 & -653 & 47 \\ \mathrm{H}(29 \mathrm{~B}) & 4640 & 4990 & -901 & 47 \\ \mathrm{H}(35) & 1716 & 3357 & 491 & 49 \\ \mathrm{H}(36) & 7056 & 4432 & 823 & 50 \\ \mathrm{H}(1 \mathrm{~A} 1) & 5247 & 3216 & 92 & 45 \\ \mathrm{H}(1 \mathrm{~A} 2) & 7268 & 3438 & 121 & 45\end{array}$

